\title{
Emergence of complex socioeconomic networks driven by individual and collective interests
}

\author{
Jaime Iranzo $\odot,{ }^{1,2}$ Federico Pablo-Martí $\odot,{ }^{3}$ and Jacobo Aguirre $\odot^{4,5,6, *}$ \\ ${ }^{1}$ Centro de Biotecnología y Genómica de Plantas, Universidad Politécnica de Madrid (UPM)-Instituto Nacional de Investigación y \\ Tecnología Agraria y Alimentaria (INIA), Madrid, Spain \\ ${ }^{2}$ Institute for Biocomputation and Physics of Complex Systems (BIFI), University of Zaragoza, Zaragoza, Spain \\ ${ }^{3}$ Complex Systems in Social Sciences Group, Universidad de Alcalá, 28801 Alcalá de Henares, Madrid, Spain \\ ${ }^{4}$ Grupo Interdisciplinar de Sistemas Complejos (GISC), Madrid, Spain \\ ${ }^{5}$ Centro Nacional de Biotecnología, CSIC. c/ Darwin 3, 28049 Madrid, Spain \\ ${ }^{6}$ Centro de Astrobiología CSIC-INTA, Ctra de Ajalvir km 4, 28850 Torrejón de Ardoz, Madrid, Spain
}

(Received 12 March 2020; revised 2 August 2020; accepted 11 November 2020; published 9 December 2020)

\begin{abstract}
The basic mechanisms that lead to the creation of complex socioeconomic structures remain poorly understood. A combination of network science and game theory can help explain them by examining how simple networks interact to build complex entities, both when connections among individuals are exclusively guided by self-interest or when they result from a mixture of individual and collective motivations. Here we present a theoretical framework where individuals or human groups from different communities connect to each other only if they increase their own eigenvector centrality, a topological measure of wide applications in many different contexts that quantifies the importance of a node within the network. Our analytical and numerical results show that the emergence of interconnected networks is catalyzed by the self-interest of peripheral agents, who are penalized in the long run but transiently benefit from establishing links with nodes from other communities. Moreover, the interconnection process leads to a hierarchical, assortative, and very efficient structure where links across networks involve nodes of the same importance. These findings are robust to the introduction of moderate levels of collective-oriented behavior and compatible with the interconnection dynamics observed in real-world socioeconomic networks.
\end{abstract}

DOI: 10.1103/PhysRevResearch.2.043352

\section{INTRODUCTION}

Throughout human history, social groups have competed for resources, knowledge, and technology, just as animals compete for food or mates in nature and firms fight for customers and production factors in modern economies. The interaction among small communities has permitted the appearance of larger and more organized structures able to more efficiently face the challenges that arise along the way. However, the undesirable consequences of social hierarchy and economic inequality have become extremely hard to overcome [1-3]. Despite the attention that this subject has attracted during the last decades [4-8], the basic mechanisms that lead to the creation of complex societies and define their internal structural properties are still a key open question.

The connection between complex networks and social sciences arose almost a century ago with the development of the concept of the sociogram [9]. However, for a long time

\footnotetext{
*Author to whom correspondence should be addressed: jaguirre@cab.inta-csic.es

Published by the American Physical Society under the terms of the Creative Commons Attribution 4.0 International license. Further distribution of this work must maintain attribution to the author(s) and the published article's title, journal citation, and DOI.
}

its use was limited and circumscribed to the fields of sociology [10,11], psychology [12], and anthropology [13,14]. It has only been in the last two decades that network theory has become popular in other areas [15] such as economics [16-19], political science [20-22], or archeology $[23,24]$. The application of network theory to social sciences has not been free of criticism, though, mostly because its potential difficulty to adequately reflect the underlying theory $[25,26]$. Nevertheless, such criticism has been losing weight as more sophisticated network-based approaches have demonstrated their power to provide precise measurements and inspire the development of new theory for a broad range of social phenomena [27,28]. Game theory has received similar criticism, especially regarding its ability to address the emergence of pro-social behaviors, such as cooperation and altruism, which are central to the development of human societies [29-31]. Although game theory has considered the importance of cooperative behaviors since its inception [32], it has developed a certain bias toward noncooperative scenarios, most notably in the field of economics, and therefore the full application of this theory to social sciences is still in progress [33].

Those caveats aside, since Axelrod's pioneering work on the emergence of cooperation between selfish agents [34], important advances have been made in the capacity of network science and game theory to explain the rise and evolution of complex socioeconomic structures [31,35-42]. In the last 
decade, the scope of these efforts has broadened to include more realistic scenarios in which networks, rather than single nodes, interact and compete at different scales, giving rise to what is usually known as a network of networks [37,43-46]. The present paper expands on this line of research.

We introduce a theoretical model aimed at describing the process by which the interconnection of small communities gives rise to complex socioeconomic structures. The process is driven by the local behavior of the members of different networked communities that contact each other exclusively guided by self-interest or following a combination of individual and collective motivations. More precisely, we consider a scenario in which groups and individuals try to maximize their own importance - prestige, wealth, or influence, depending on the case-with respect to other groups and individuals. As a quantitative proxy for importance, we adopt a topological measure, the eigenvector centrality [47], whose applicability to socioeconomic systems is presented in Sec. II.

Our analytical and numerical results show that the interconnection among communities is facilitated by the action of their weakest members, whose fate is often decoupled from that of their own community. In contrast, central nodes, whose well-being aligns better with the collective fate, only engage with the interaction process when it has become inevitable. The emerging process leads to an assortative society-i.e., nodes connect to other nodes of the same importance, a typical property in social structures [48]—which is very efficient in dynamical terms such as the growth rate or the dispersion time in spreading processes. Paradoxically, inequality diminishes during the process but is recovered when the new interconnected network reaches its asymptotic structure. As a descriptive example, we simulated the model on networks of rural communities from southern India [49-51], and assessed its applicability to a wide range of real systems, focusing on the spread of scientific collaborations in graphene research from 2007 to 2018 and the analysis of pairing in the context of religious, social, or ethnic groups, as well as in international business activities.

\section{THE EIGENVECTOR CENTRALITY IN SOCIOECONOMIC SYSTEMS}

Eigenvector centrality measures the importance of a node or group of nodes in a network based on the relevance of its neighbors [47]. Its value is obtained from the eigenvector associated with the largest eigenvalue of the adjacency matrix. In practice, the eigenvector centrality describes the power of a node to affect other nodes in the network not only directly but also indirectly through its neighbors [52]. For example, in the context of scientific collaborations, a scientist linked to colleagues with high eigenvector centrality will also show high centrality. Note, however, that while being connected to few but very important nodes is a guarantee of large eigenvector centrality, this is not necessarily true when connecting to many but not so relevant neighbors.

This topological measure can be applied to most processes whose final state is related to the spectral properties of the network, such as the spread of knowledge, wealth, innovation, culture, rumors, or diseases [53]. Some applications to very diverse contexts are the study of the impact of a scientists' work in the community [54], the webpage ranking in the internet through the Pagerank algorithm [55], the evolution of genotypes [56,57], and the detection of relevant regions in the brain [58], to cite just a few.

Focusing on the socioeconomic literature, eigenvector centrality has received special attention as a proxy for the influence or prestige of an individual or human group embedded in a community [59,60], the systemic relevance and robustness of firms and institutions [61], and the amount of money, goods, and services in economic and financial networks [62-65]. In this context, the adequacy of eigenvector centrality as a measure of a node's importance is supported by multiple lines of evidence. For example, a recent study found that voluntary enrollment into a microfinance program in rural India significantly increased when the program was introduced through locals with higher eigenvector centrality because their influence in the community was larger [50,51]. Furthermore, the correlation of eigenvector centrality with the systemic relevance and robustness of firms and institutions embedded in financial networks has been extensively studied, both theoretically and empirically, and it is linked to greater opportunities for future development and better access to reliable credit sources in the event of a financial crisis [61,66-68]. In other cases, eigenvector centrality represents valuable knowledge for the production of a good or the control of a technique for which individuals or companies compete [62,69-71]. Accordingly, this topological measure has proved especially fruitful to study knowledge spreading in interfirm networks and to identify those companies that act as sources of innovation [72,73]. A similar effect has been described in research collaboration networks, where eigenvector centrality constitutes a good predictor of article and patent productivity [74].

Some works have studied the evolution of networks when creation and destruction of links is based on several measurements of node centrality. In single networks, centrality-based rewiring leads to nested structures, where the neighborhood of every node is a subset of the neighborhoods of nodes with larger degree [70,75]. It is worth mentioning, though, that most of these works focus on single networks, and it is well established that many of the properties of isolated (single) networks drastically differ from those of interconnected networks $[45,64,76]$. More importantly, intra- and intercommunity links in real networked systems often differ in properties and dynamics [77], which warrants a dedicated study of centrality-based dynamics in networks connected to other networks. A first step in that direction was presented in Refs. [37,76], where competition for eigenvector centrality at the whole-network level was shown to determine the largescale configuration of connections across networks. In turn, here we focus on interconnections at the level of individual nodes, as our aim is to analyze how different distributions of individual and collective interests drive the emergence of interconnected socioeconomic networks.

\section{THE EMERGING SOCIETY GAME}

In our model, we consider $r$ networks of $N_{i}$ nodes respectively, where $i=\mathrm{A}, \mathrm{B}, \mathrm{C}, \ldots$ The adjacency matrices $\mathbf{G}_{i}$ contain full information about the connections (intralinks) 
within each network $i$, that is, the specific topology of the networks. The largest eigenvalue $\lambda_{i}$ of $\mathbf{G}_{i}$ grows with the number of nodes and links, and serves as a proxy for the network strength (depending on the context, this could mean the rate at which knowledge or wealth is generated) [76]. Networks can be sorted from strongest to weakest, such that $\lambda_{\mathrm{A}} \geqslant \lambda_{\mathrm{B}} \geqslant \ldots \geqslant \lambda_{r}$. Collectively, the $r$ networks constitute the total network $\mathrm{T}$, with $N_{\mathrm{T}}=\sum_{i} N_{i}$ nodes. The adjacency matrix $\mathbf{G}_{\mathrm{T}}$ includes the intralinks that belong to each network and the links among nodes from different networks (henceforth connector links). In this framework, intra- and intercommunity connections can share the same general nature (e.g., friendship, trust, commercial partnership) but evolve at different rates, as we assume that complex societies are built upon the foundations of already existing smaller structures. In practice, we maintain the internal structure of each network and only the connector links evolve with time.

In the simplest form of the game, the goal of each node $n$ is maximizing its own importance quantified as its eigenvector centrality $C_{n}$, calculated as

$$
C_{n}=\frac{\left(\vec{u}_{\mathrm{T}}\right)_{n}}{\sum_{j=1}^{N_{\mathrm{T}}}\left(\vec{u}_{\mathrm{T}}\right)_{j}},
$$

where $\vec{u}_{\mathrm{T}}$ is the eigenvector associated with the largest eigenvalue $\lambda_{\mathrm{T}}$ of the adjacency matrix of the total network. The game starts with all networks isolated from each other. In such disconnected configuration, only the nodes in the strongest network acquire positive centrality, while the centrality of all other nodes is zero. In each time step, two random nodes belonging to different networks (henceforth connector nodes), for example, $l \in \mathrm{A}$ and $m \in \mathrm{B}$, are connected by an undirected connector link of weight $\epsilon$. For simplicity, only one connector link per node is allowed, and therefore any preexisting connector links associated to nodes $l$ or $m$ are erased (but note that accepting a larger, but still small number of connections per individual does not qualitatively affect the results). If both nodes are already connected with each other, the link is erased, allowing the deletion - not only rewiringof already existing connector links. The new configuration is accepted only if both connector nodes $(l$ and $m)$ increase their centrality; otherwise, the system returns to its former state. Note that connector links affect the centrality of every node in every network because the eigenvector centrality is a global quantity; however, the intrinsic strengths $\lambda_{i}$ of the networks remain unchanged as long as connector links involve nodes from different networks (as is the case here).

The system can remain in the initial disconnected state indefinitely (if there is no possible connector link that simultaneously benefits two connector nodes belonging to different networks) or it can experience a complex evolutionary process involving creation, rewiring, and destruction of connector links. Regardless of the particular random steps of this process, the game ends when a Nash equilibrium is reached [78] such that pairs of nodes cannot increase their centrality anymore by locally rewiring their connector links.

Because the overall centrality of the total network is always equal to 1 [Eq. (1)], any competition for centrality among networks is a zero-sum game. This has important implications for the emerging society game. In the initial disconnected state, all centrality is accumulated in the strongest network, and any connection with weaker communities diverts centrality toward those. Thus, from a community perspective, early connections are detrimental to the strong network and the creation of connector links is only possible if the benefit that the connector node obtains by acquiring a new contact outweighs the loss of centrality experienced by its community. Nodes in weak networks, however, do not face this dilemma because connecting to the strong network at the initial step is beneficial for both the whole community and the individual nodes. In summary, the interaction of simple ensembles toward larger and more complex structures will only be feasible through the self-interest of individuals that act regardless of the fate of their own community.

\section{INTERCONNECTION DYNAMICS DRIVEN BY SELF-INTEREST}

\section{A. Interaction between two networks}

To simulate the dynamics of the emerging society game in real-world networks, we used survey data obtained in the context of a microfinance program in rural communities from southern India [49-51]. We built the loan networks of these communities by connecting individuals that were willing to lend and borrow a certain amount of money from each other. Local loan networks constructed this way provide valuable information about the financial resilience of a region [79-82], how much neighbors trust each other, and with whom the villagers wouldn't mind sharing a technical innovation or a new tool. We use these real-world networks as an illustrative starting point to describe the phenomenology of a generic interconnection process in which agents connect to each other pursuing greater centrality. Note, however, that competition for centrality in this example could result from the households' drive to optimize financial resilience or to increase their influence over the community, as already discussed in Sec. II. In fact, Banerjee et al. showed in Ref. [50] that the eigenvector centrality calculated from these networks was the measure that best quantified the influence of a villager on the community, outperforming other topological quantities such as the degree centrality or betweenness.

Figure 1 shows a simulation of the emerging society game on two of the Indian village networks--note that the same phenomenology is obtained when the model is applied to random Erdős-Rényi networks (see Fig. 1 of the Supplemental Material [83]) and when two connector links per node are allowed (see Fig. 2 of the Supplemental Material [83]). In the beginning, the villages are isolated and all centrality is assigned to the village with the strongest network (henceforth network A). After a certain amount of creation, rewiring and deletion of connector links [Fig. 1(a)], the two villages reach a state of equilibrium characterized by a high number of connections [Fig. 1(b)]

Three stages spontaneously emerge during the process before arriving to the asymptotic regime: (i) the approaching stage, (ii) the connecting stage, and (iii) the optimization stage. In the approaching stage, there exists a critical connecting centrality $\left(\vec{u}_{A}\right)_{\text {crit }}$ such that only those nodes in A with cen- 

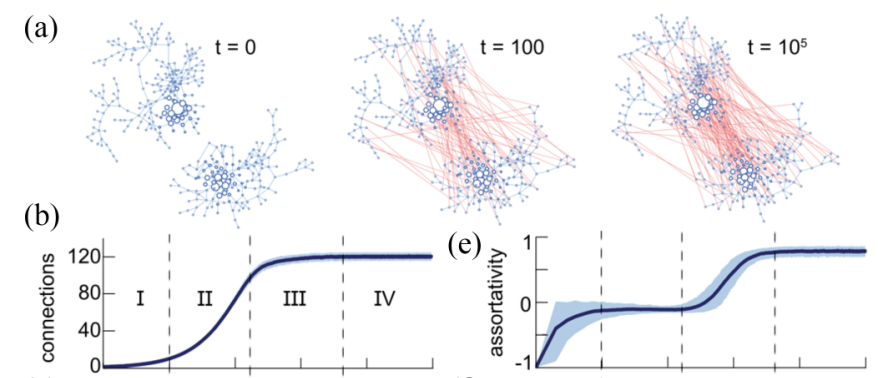

(c)
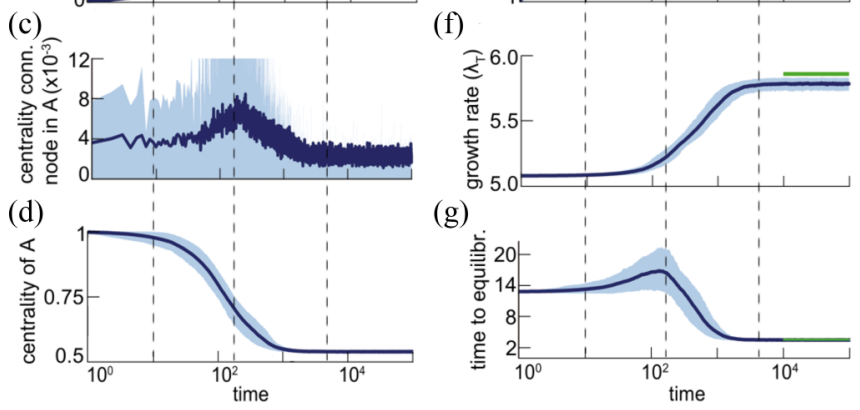

FIG. 1. Numerical simulation of the emerging society game: Two initially isolated networks evolve toward a larger and more complex structure (networks 60 and 44 of Ref. [49], with 213 and 138 nodes, respectively; $\lambda_{\mathrm{A}}=5.0785, \lambda_{\mathrm{B}}=4.6961$ ). Dark lines (shadowed areas) correspond to averages (10\%-90\% percentile) over 200 realizations. Only accepted connections are plotted. The strength of the connector links is $\epsilon=1$ in all simulations, unless specified otherwise. (a) The state of the system at three different times. (b) Number of connector links between both networks. (c) Centrality of the most recent connector node from network A. (d) Centrality accumulated by network A. (e) Assortativity of connections, measured as the correlation coefficient of the connector node centralities (for consistency, here we used time-independent node centralities, separately measured in each network). (f) Largest eigenvalue $\lambda_{\mathrm{T}}$. (g) Time to equilibrium $t_{\mathrm{eq}}=\left[\ln \left(\lambda_{\mathrm{T}} / \lambda_{\mathrm{T}, 2}\right)\right]^{-1}$, a quantity proportional to the average time to reach the equilibrium in a generic spreading process [56]. The maximum reachable growth rate and minimum time to equilibrium (marked in green) were determined by numerically optimizing these quantities subject to the constraint of a single connector link per node. Stages of the process: I, approaching; II, connecting; III, optimization; IV, equilibrium stage.

trality smaller than that critical value will connect to network B. For the first connector link, we obtain

$$
\left(\vec{u}_{A}\right)_{\text {crit }}=\sqrt{\frac{2 \Delta \lambda}{\lambda_{A}}},
$$

where $\Delta \lambda=\lambda_{A}-\lambda_{B}$ is the difference between the strengths of both networks. This critical value is especially low and therefore hinders the connection between networks, when the largest eigenvalues of both networks are very similar. The following connector links further decrease the critical centrality toward

$$
\left(\vec{u}_{A}\right)_{\text {crit }}=\sqrt{\frac{\Delta \lambda}{\lambda_{A}}}
$$

(see Appendix $\mathrm{C}$ for the formal proof of these expressions and the rest presented in this section). Thus, during these first steps, connector links are created exclusively between the most peripheral nodes of network A [the underdogs of the strong village, see Fig. 1(c)] and any node of network B, because of the reluctance of central nodes in the strong network to lose importance and the tendency of all nodes of the weak network to connect to a promising source of centrality. As a result, the system becomes dissortative in this first stage and the average centrality or importance of the peripheral nodes increases.

The connecting stage starts as just mentioned, with a few peripheral nodes in A connected to all types of nodes in B. Nodes in B already connected to a (peripheral) old node in A can now reconnect to a new (so-far disconnected from B) node in A as far as the centrality of this new node is bounded as follows:

$$
\left(\vec{u}_{A}\right)_{\text {new }} \in\left(\left(\vec{u}_{A}\right)_{\text {old }},\left(\vec{u}_{A}\right)_{\text {crit }}\right) .
$$

$\left(\vec{u}_{A}\right)_{\text {crit }}$ is given by the complex expression shown in Eq. (C8) of Appendix C, but when the number of connector links grows sufficiently, it tends to

$$
\left(\vec{u}_{A}\right)_{\text {crit }}=\frac{\left(\vec{u}_{A}\right)_{\mathrm{old}}}{2}+\sqrt{\frac{\Delta \lambda}{\lambda_{A}}+\left[\frac{\left(\vec{u}_{A}\right)_{\mathrm{old}}}{2}\right]^{2}} .
$$

That is, at the beginning of the connecting stage, nodes in $\mathrm{B}$ that are already connected to peripheral nodes in A will rewire their connections toward slightly more central nodes in A. The critical centrality $\left(\vec{u}_{A}\right)_{\text {crit }}$, that was around $\sqrt{\frac{\Delta \lambda}{\lambda_{A}}}$ at the end of the approaching stage and did not let nodes of larger centrality connect to $\mathrm{B}$, increases up to a quantity that grows with $\left(\vec{u}_{A}\right)_{\text {old }}$ [see Eq. (4)]: Each time a new node in A of centrality larger than the rest of connected nodes steals a link from a weaker node in A, the critical centrality also grows, permitting the connection of even more central nodes. Little by little, a staircase of more central nodes in A enters the game [Fig. 1(c)], diverting centrality from network A to network B [Fig. 1(d)]. After a large number of connections and rewirings, the majority of nodes in $\mathrm{A}$ and $\mathrm{B}$ become connected to the other network in a random manner, reaching the end of the connection stage. In this transition point, the system reaches a maximum of disorder, which manifests as a minimum absolute value of assortativity [Fig. 1(e)], and a maximum in the time that it takes for the system to recover from a generic perturbation [Fig. $1(\mathrm{~g})]$.

In the subsequent optimization stage, the gain of centrality of a node $l$ in A that is connected to an old node in B and rewires its connection toward a different new node in $\mathrm{B}$ verifies

$$
\Delta C_{l \in A} \propto\left(\vec{u}_{B}\right)_{\text {new }}-\left(\vec{u}_{B}\right)_{\text {old }}
$$

In a similar manner, for nodes $m$ in $\mathrm{B}$, we obtain

$$
\Delta C_{m \in B} \propto\left(\vec{u}_{A}\right)_{\text {new }}-\left(\vec{u}_{A}\right)_{\text {old }},
$$

[see Eqs. (C10) and (C11) of Appendix C for details]. This yields that every node will do its best to avoid detrimental connections with weaker nodes and to connect to more central nodes. The process leads to a hierarchical equilibrium state (a Nash equilibrium), where nodes end up linking to nodes of its same importance, optimizing the assortativity of the system (measured only in the connector links, as the internal links of 
both networks are kept constant throughout the process). Also, the largest eigenvalue of the total network reaches a maximum close to the optimum [Fig. 1(f)], and the time to equilibrium (or recovery time) decreases toward its potential minimum [Fig. 1(g), see Appendix D for a mathematical proof]. As a consequence, the final structure-the union of networks A and $\mathrm{B}$ - is optimally efficient in dynamical terms, as influence, wealth, knowledge, or whatever resource we are measuring in the process will grow at its maximum rate and the spread of information and goods across the network will be optimally fast. Note, however, that the maximum assortativity reached at the end of the interconnection process will push the peripheral nodes again into the background, with most of them disconnected from any node of the other network.

Besides the analytical information sketched above, a thorough theoretical study of the interconnection process is developed in the Appendices. In Appendix A, we present a full mathematical treatment of the emerging society game, whereas in Appendix B we assess the good agreement between the numerical and analytical approaches. In Appendix $\mathrm{C}$, we formally describe all three stages of the process and the transitions between them and we prove that the asymptotic state of the system is a Nash equilibrium that maximizes assortativity. Finally, in Appendix D, we prove that the final equilibrium optimizes the dynamical properties of the system.

\section{B. Interaction among many networks}

What would happen if more than two communities interacted to become a much larger structure? To address this question, we modeled the connection dynamics of nine networks with three different topologies (Barabási-Albert scale-free, Erdős-Rényi random, and a group of villages from the microfinance program [49-51]). In all cases, we found that the same phenomenology described above for the twonetwork system also occurs in many-network systems (see Figs. 3-5 of the Supplemental Material [83]). In Fig. 2, we study the connection process at the network-scale, that is, we consider networks as the interacting units and focus on the number of interconnections between pairs of networks, rather than on the properties of the connector nodes. At the beginning of the process (the approaching stage), all networks (and specially the weakest) tend to connect to the strong one, as this is the only source of centrality, and the larger the difference between eigenvalues the easier the connection [see Eqs. (2) and (3) and Appendix C for details]. At the connecting stage, the system faces a disordered configuration where connector links are randomly distributed among the networks and, after the optimization stage, strong networks link with each other in a rich club that accumulates most of the final centrality and ignore the weakest neighbors. In summary, the phenomenology observed in the two-network system is also detected in multinetwork systems and, interestingly, the behavior shown at the node scale is mimicked at the network scale.

\section{INDIVIDUAL VERSUS COLLECTIVE MOTIVATIONS}

It is known that in some social and economic networks, the decisions of the individuals are based on both group and individual motivations. Following Ref. [84], we redefined the centrality of an individual $n$ as a mixture of collective and individual interests, such that

$$
C_{n}^{*}=\alpha C_{n}+(1-\alpha) \frac{\sum_{k=1, k \neq n}^{N_{i}} C_{k} \beta^{d_{n, k}-1}}{\sum_{k=1, k \neq n}^{N_{i}} \beta^{d_{n, k}-1}},
$$

where $\alpha \in[0,1]$ is the self-interest parameter, $\beta \in[0,1]$ the normalized social reach, and $d_{n, k}$ is the distance from node $n$ to a node $k$ of its own network $i=\mathrm{A}, \mathrm{B}, \ldots$ The raw centralities of node $n, C_{n}$, and of node $k, C_{k}$, are given by Eq. (1).

The self-interest parameter $\alpha$ can be interpreted as the fraction of the total payoff of an individual that is due to its own activity or initiative. $1-\alpha$ could be the side benefits that an agent receives for belonging to a community or the incentive by which the authorities promote community-oriented strategies, depending on the case [Fig. 3(a), top]. According to this, $\alpha=1$ when the payoff is exclusively that of the connector node individually - the case studied in the former section-and $\alpha=0$ when the payoff coincides with that of the neighborhood with which it shares the benefits of its actions. The normalized social reach $\beta$ tunes the size of such social group, and $\rho=1-1 / \log (\beta)$ is its average radius [Fig. 3(a), bottom]. $\beta=0$ (i.e., $\rho=1$ ) represents the case in which the nodes are only involved with the fate of their neighbors at distance 1 and do not care about the rest, while $\beta=1$ (i.e., $\rho=\infty$ ) when all nodes in the network are equally important for each agent.

Figure 3(b) shows that the outcome of the interaction between two networks critically depends on the values of $\alpha$ and $\beta$. Remarkably, there is a drastic transition between the cases in which the networks do not ever connect and those in which the whole connecting process takes place. In the limit when $\alpha=0$ and $\beta=1$, the payoff of every individual coincides with that of its whole network (excluding itself). In such a limit case, it is easy to see that the system will remain disconnected, because an isolated strong network always loses centrality if it connects to any other network.

Figures 3(c)-3(h) plot the dependency of the critical values $\alpha_{\text {crit }}$ (for $\beta=0$ ) such that the connecting process only takes place for $\alpha>\alpha_{\text {crit }}$ and $\beta_{\text {crit }}$ (for $\alpha=0$ ) such that the connecting process only takes place for $\beta<\beta_{\text {crit }}$ on some key properties of the networks, and Fig. 6 of the Supplemental Material [83] shows the dependency of $\alpha_{\text {crit }}$ on the centrality of the connector nodes of the strong network (see Appendix E for an analytic calculation of $\alpha_{\text {crit }}$ and a formal analysis of its dependency on the network properties). Numerical and theoretical results agree and demonstrate that $\alpha_{\text {crit }}$ is remarkably low-i.e., little self-interest would be enough for initiating the connecting process, except if the strength $\lambda_{\mathrm{A}}$ of the strong network is very large, if both networks show very similar eigenvalues, or if the centrality of the most peripheral nodes is large, which only happens when the networks are small and their structure is regular or cliquelike. Furthermore, the dependency of $\alpha_{\text {crit }}$ and $\beta_{\text {crit }}$ on the network properties is qualitatively inverse: The connecting process will only take place if the individuals act sufficiently in their own interest or if they are altruistic but share their payoff with a community that is small and close to them. 

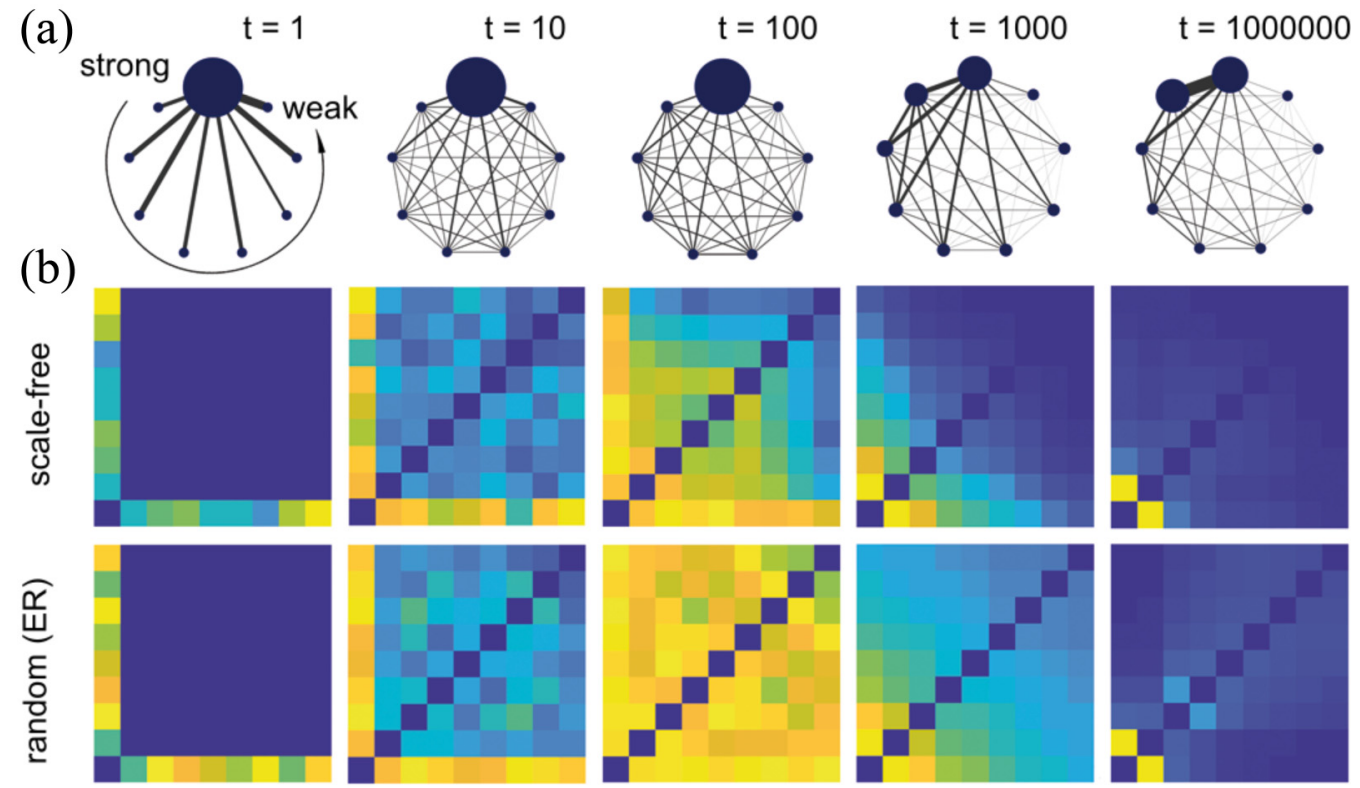

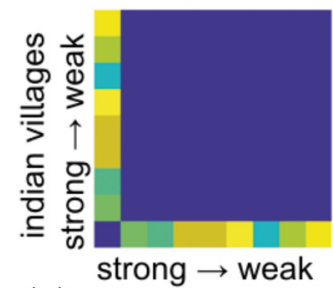

(c)

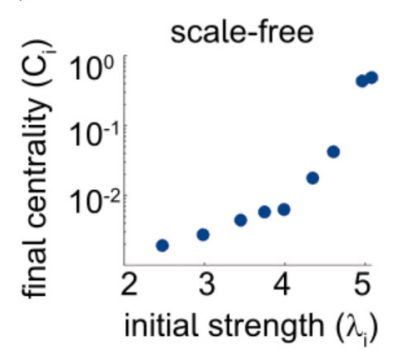

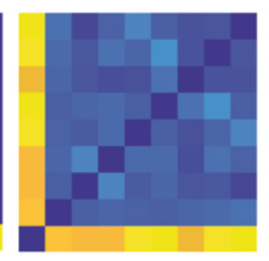
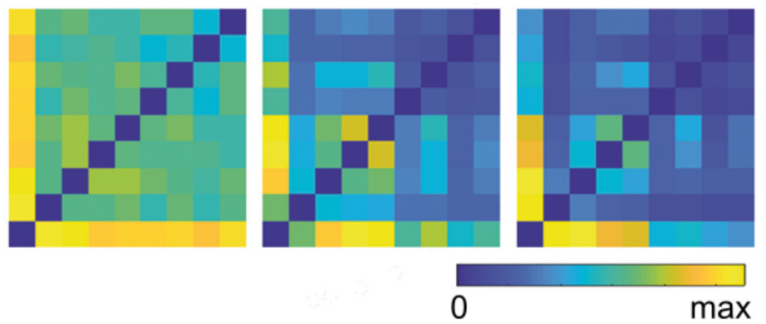

random (ER)
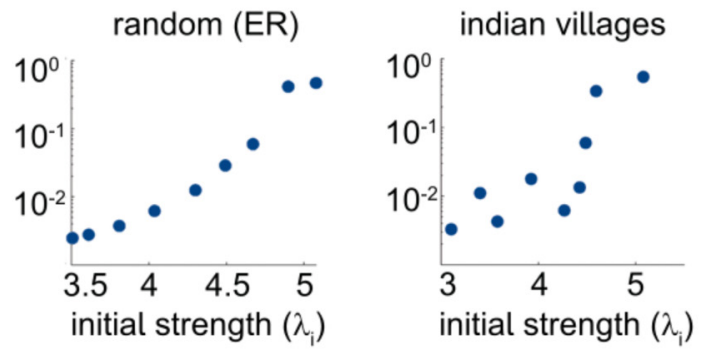

FIG. 2. Numerical simulation of the emerging society game in a set of nine interacting networks. One hundred simulations were carried out using scale-free (Barabási-Albert) networks (whose connection patterns are sketched in (a)), random (Erdős-Rényi) networks, and real loan networks from Indian villages [49]. The networks were ranked according to their strength (i.e., maximum eigenvalue $\lambda_{i}$ ). The number of connector links between each pair of networks (plotted in (b) at different times) shows that the three stages of the connection process detected in the two-network case also occur when many networks play the game. (c) When the equilibrium state has been reached, the final centrality accrued by the nodes of each network $\left(C_{i}\right)$ correlates with the network's initial strength $\left(\lambda_{i}\right)$. Note that in Ref. [37] it was shown that if the interconnection between the weak networks reaches a larger maximum eigenvalue than that of the strongest network, several final configurations might coexist, complicating the analysis of the system. The systems studied here are not the case though.

\section{ANALYSIS OF REAL-WORLD SOCIOECONOMIC SCENARIOS}

\section{A. A real system studied along time: scientific collaborations in an emergent field of research}

The unprecedented access to bibliographic databases linking research funding, scientific productivity, and scientific collaboration patterns has made science of science [86] a promising research area, which could help accelerate the development of science by more effectively addressing environmental, social, and technological challenges, and by facilitating the identification of the mechanisms responsible for scientific discovery [87].
To test if the evolution of scientific collaborations follows the steps predicted by our model, we studied the spread of collaborations in the field of graphene from 2007 to 2018 . We chose this field because, while being relatively new, it has experienced rapid growth and is already well established in the physics community.

We first investigated whether the structure of the collaboration network can predict the impact of a scientist's work. To that end, we collected the h-index of the hundred most central researchers in the graphene coauthorship network in May 2020 and carried out a stratified analysis to assess how a researcher's centrality (measured over the period 2007-2018) and the network community to which that researcher belongs 
(a) self-interest<smiles>OOC1CC(OO)C(O)O1</smiles>

social reach

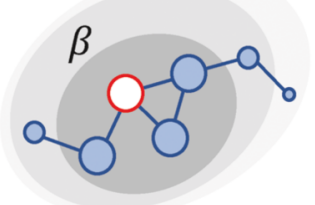

(b)

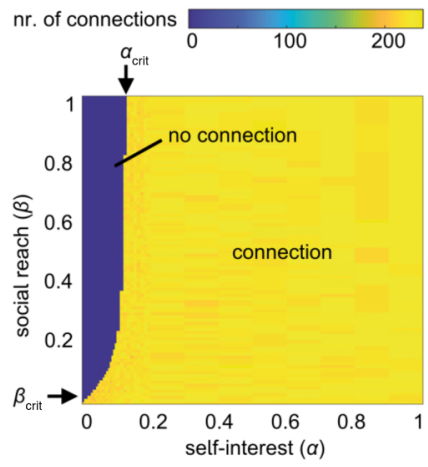

(c)

口 SF, $\lambda_{\mathrm{B}} / \lambda_{\mathrm{A}}=0.8 \quad \mathrm{SF}, \lambda_{\mathrm{B}} / \lambda_{\mathrm{A}}=0.95 \quad \circ \mathrm{ER}, \lambda_{\mathrm{B}} / \lambda_{\mathrm{A}}=0.8 \quad \bullet \mathrm{ER}, \lambda_{\mathrm{B}} / \lambda_{\mathrm{A}}=0.95$

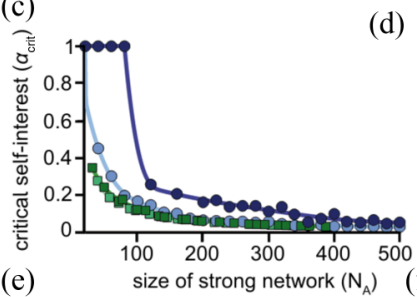

(e) size of strong network $\left(\mathrm{N}_{\mathrm{A}}\right)$ (f)

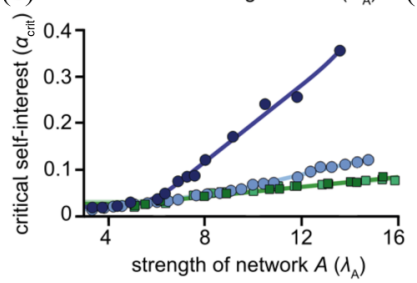

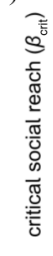
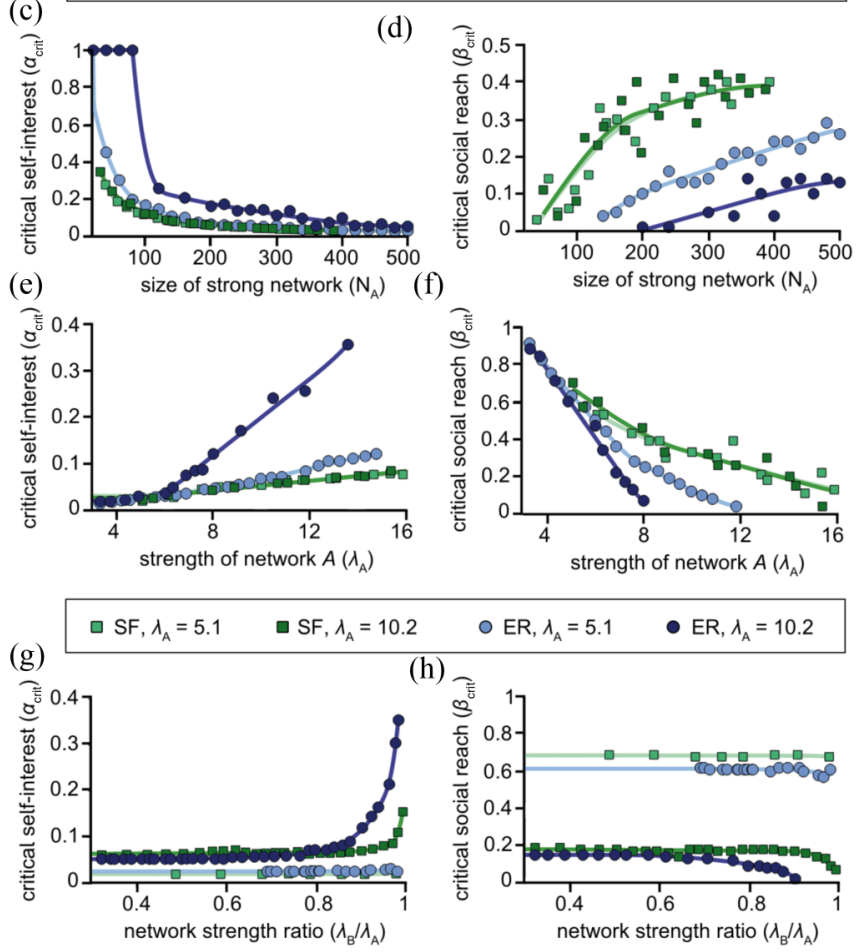

(h)

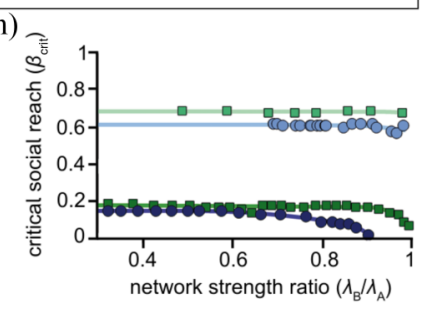

FIG. 3. Individual versus collective motivations in the emerging society game. (a) The self-interest parameter $\alpha$ weights the relative contribution of individual and collective motivations to the centrality of an individual; the normalized social reach $\beta$ tunes the radius of collective motivations. (b) Number of connections at equilibrium between two Erdős-Rényi networks as a function of $\alpha$ and $\beta$. Connection occurs if $\alpha>\alpha_{\text {crit }}$ (for $\beta=1$ ) or if $\beta<\beta_{\text {crit }}$ (for $\alpha=0$ ). (c)-(h) Dependency of $\alpha_{\text {crit }}$ [(c), (e), (g)] and $\beta_{\text {crit }}$ [(d), (f), (h)] on the size of the strong network $N_{\mathrm{A}}[(\mathrm{c}),(\mathrm{d})]$, the maximum eigenvalue of the strong network $\lambda_{\mathrm{A}}[(\mathrm{e}),(\mathrm{f})]$, and the ratio of eigenvalues $\lambda_{\mathrm{B}} / \lambda_{\mathrm{A}}$ $[(\mathrm{g}),(\mathrm{h})]$, calculated in networks of different sizes and topologies (ER: Erdős-Rényi, SF: scale-free). Unless otherwise specified, $\lambda_{\mathrm{A}}=$ $10.2, N_{\mathrm{A}}=213$.

reflect on his/her h-index. We found that both the centrality and the community contribute to the scientist's impact. The quantitative contribution of both factors is similar, with the community explaining $25 \%$ of the variance in the $h$ index (ANCOVA, $F=2.7,13$ d.f., $p=0.004$ ) and centrality explaining an additional $20 \%$ (ANCOVA, $F=28.4$, 1 d.f., $p<10^{-5}$ ). After correcting for the effect of the community, (a)
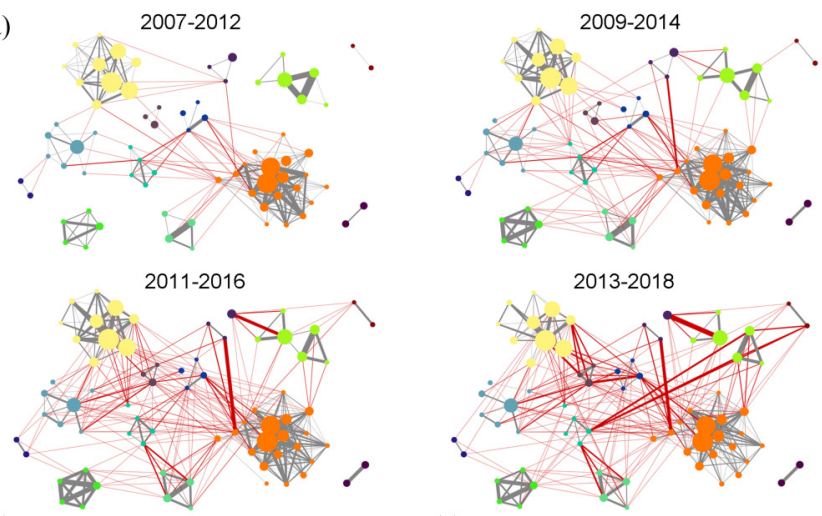

(b)

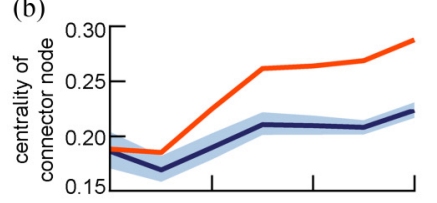

(d)

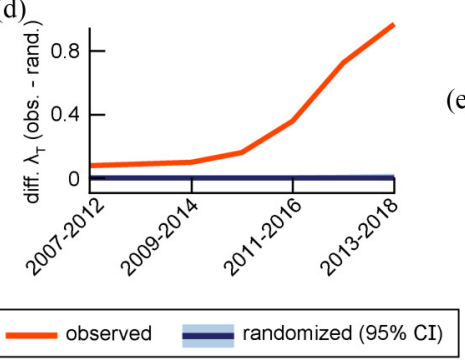

(c)
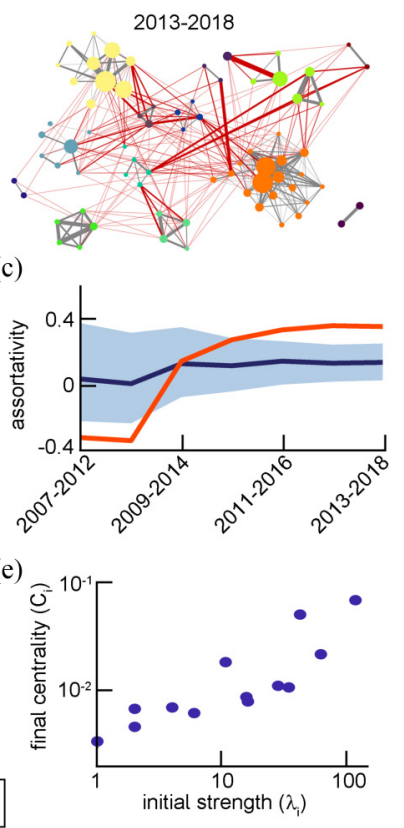

FIG. 4. Evolution of the scientific coauthorship network in the field of graphene from 2007 to 2018. 104670 articles and 193675 scientists were used in the study (for clarity, only the top 100 researchers are represented). (a) Scientific collaboration networks in five-year windows starting in 2007, 2009, 2011, and 2013. Nodes represent researchers, with node sizes proportional to their eigenvector centrality. Node colors indicate the communities of the original network (2007-2012) detected by Infomap [85]. Link widths are proportional to the number of coauthored articles (connector links in red). (b) Mean centrality of the connector nodes. (c) Assortativity, measured as the correlation coefficient of the connector nodes' centralities. (d) Difference between the maximum eigenvalue (growth rate) of the system and that of the same communities interconnected through a randomized set of connector links. (e) Dependence of the final centrality of each network $C_{i}$ on the network's initial strength $\lambda_{i}$ (Spearman's correlation coefficient $\rho=0.91, p<10^{-4}, n=13$ ). In (b)-(d), blue lines (shaded areas) indicate mean values $(95 \%$ confidence intervals) for 100 randomizations of the connector links. See Appendix F for details.

the correlation coefficient between centrality and h-index becomes $R=0.52$ (see Fig. 7 of the Supplemental Material [83], $N=98, p<10^{-7}$ ).

Having shown that the structure of the collaboration network is relevant to the midterm impact of an author's research, we focused on the dynamics of such collaborations over time. Figure 4(a) shows the connections for the hundred most central researchers in five-year periods (internal links in gray and connector links in red). In agreement with the model, the connector links in 2007 mostly involved peripheral nodes, whereas long-reach collaborations among central researchers were established in subsequent years [Fig. 4(b)]. Furthermore, 
the shift from negative to positive assortativity already seen in the simulations [Fig. 1(e)] is also observed in this real case [Fig. 4(c)]. Figure 4(d) presents the increase in the maximum eigenvalue (growth rate) of the system in comparison to the same communities when connected through a randomized set of connector links. Finally, Fig. 4(e) shows that, at the end of the process (i.e., in the year 2018), the final centrality of each community $\left(C_{i}\right)$ correlates with its initial strength $\left(\lambda_{i}\right)$, in agreement with the examples analyzed in Fig. 2.

In summary, the patterns observed for the establishment of scientific collaborations are fully compatible with the phenomenology described in this paper: starting from a fragmented network, intergroup collaborations initially linked peripheral scientists from large groups (probably postdoctoral researchers with high mobility) with relevant professionals from smaller groups. These original connections catalyzed the collaboration among more senior scientists that, taking part in this process in a second stage, led to the current, very active, intertwined, and more hierarchical network, where most centrality accumulates in the strongest groups. The final equilibrium has not yet been reached, though, and both a decrease of the mean centrality of the researchers that establish new collaborations and a saturation of the maximum eigenvalue of the system should be expected for the following years.

\section{B. Assortativity in real socioeconomic networks}

The previous example is exceptional because it covers the evolution of an ensemble of interconnected networks from its early stages to near maturity. In contrast, most socioeconomic networks with available data represent advanced stages of the interconnection process. Although we cannot study the dynamics of those networks in as much depth as we did with graphene collaborations, we expect that they will display some general properties that are characteristic of the stationary state of the process. Specifically, we focused on two robust predictions of the emerging society game: As the final network reaches the stationary state, (i) nodes from a community connect to nodes of similar importance from other communities and (ii) interconnections tend to involve communities of similar strength. We tested these predictions in a diverse set of real-world networks that include, among others, friendship networks in African rural communities [88] and business affinity networks revealed by overlap in their boards of directors [89]. As predicted by the model and shown in Fig. 5, the networks studied are assortative both at the node level (one-sample student's $T$ test, $T=8.8$, 44 d.f., $p<10^{-10}$ ) and at the community level (one-sample student's T test, $T=19.1,38$ d.f., $p<10^{-10}$ ).

\section{Individual versus collective motivations in real socioeconomic networks}

The centrality-based framework described in this paper naturally applies to social interaction processes in which individuals aim to connect with broader environments than those where they normally operate. The drive to connect with individuals outside the group can be more or less intense depending, among other factors, on the degree of satisfac-

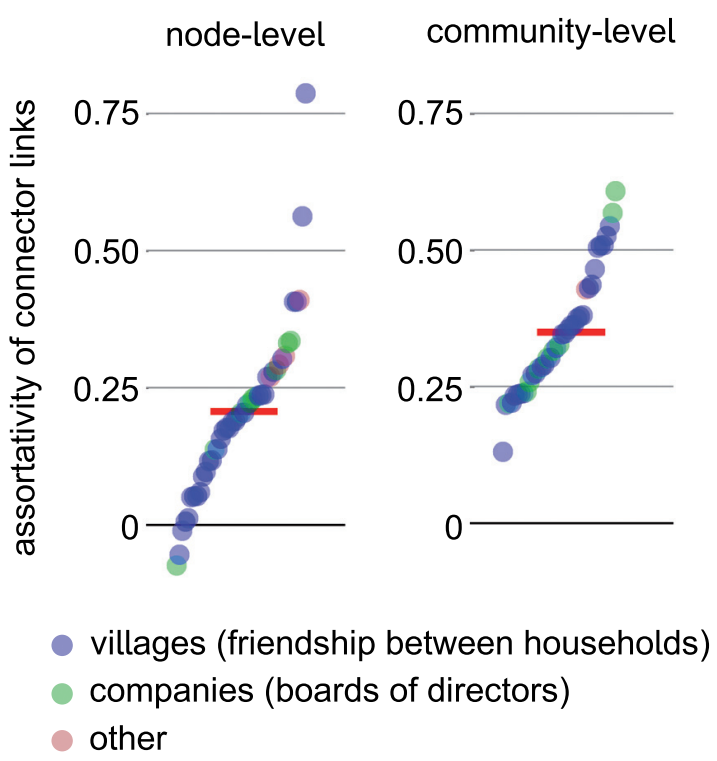

FIG. 5. Assortativity of connector links in real-world socioeconomic networks. Network communities were detected with Infomap [85] and links were classified as intracommunity or connector, depending on whether they involve nodes from the same or different communities. Intracommunity links were used to calculate the strength (i.e., the largest eigenvalue of the adjacency matrix) of each community. The node-level assortativity (left) measures the correlation between connector node centralities. The communitylevel assortativity (right) measures the correlation in the strengths of interconnected communities, weighted by the number of connector links. Horizontal red lines indicate the mean assortativities, equal to 0.21 and 0.35 at the node and community level, respectively.

tion offered by the relationships already existing within the group [90,91]. The propensity to open up to external connections is also influenced by the nature of the group; for example, many highly cohesive communities, such as religious, ethnic or even family groups, favor pairings with members of the group itself [92,93], which negatively affects the propensity to establish intergroup relationships [94,95]. In that context, the self-interest parameter $\alpha$ represents the disregard or indifference of the individual toward the loss of internal cohesion that the group will experience as a consequence of the new external connection. It is deeply related to the conformity (i.e., the force behind our desire to fit in, low $\alpha$ ) and anti-conformity (high $\alpha$ ) biases, widely studied in cultural evolution [96]. In turn, the normalized social reach $\beta$ reflects the depth of the social field that influences the individual's decision-making.

Taking these two factors into account, we can classify the cases where the model is applicable in four large categories (Fig. 6). Category I (low $\alpha$, high $\beta$ ) includes scenarios where the decision to connect is strongly influenced by the whole group (high conformity bias), therefore hindering intergroup connections. That is the case of some ethnic, cultural or religious communities [97,98] that establish barriers to pairings with individuals that do not belong to the group [99]. Category II (low $\alpha$, low $\beta$ ) is also characterized by a high conformity bias, but the reference is no longer the whole group but a closer environment, such as the family or the circle of friends 


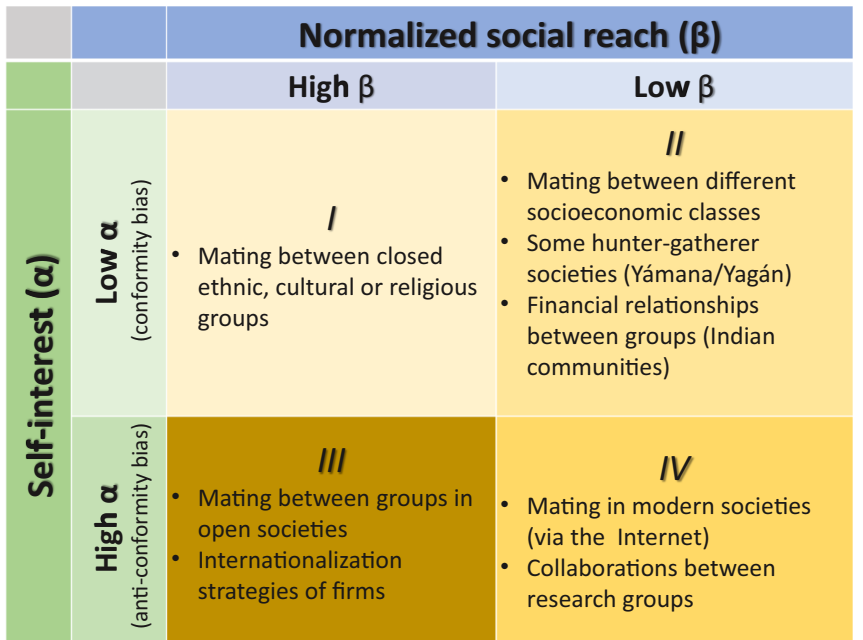

FIG. 6. A classification of real-world socioeconomic scenarios where the connection among small communities is differentially affected by individual and collective motivations.

or co-workers. Family and peer pressure against matches with people from different socioeconomic backgrounds would fall in this category [100]. Another example is found in certain hunter-gatherer societies such as the Yámana/Yagán, which develop in very limited social groups but on certain occasions, such as when a whale is stranded on the beach, meet in larger gatherings which are used to make pairings [101]. Category III (high $\alpha$, high $\beta$ ) would include societies that are open to relationships across groups or whose members reject or ignore the pressures received [102]. This is also the behavior usually followed by firms in their internationalization strategies. When looking for business partners in other countries, firms try to improve their economic results [103] although on certain occasions they may be affected by state restrictions such as tariffs or quotas. Finally, in category IV (high $\alpha$, low $\beta$ ), the decision to connect is mainly due to self-interest, but when the interests of the group are taken into account, it is usually with those who are closest. Pairings via the internet provide a clear example of this category: through the web, individuals seek to find their ideal partner out of the physical group to which they belong, even though this could affect the dynamics among family or friends [104]. Category IV also includes scientific collaborations, which are mainly driven by the researchers' aim to improve their position and the scope of their research, but can sometimes take into consideration the effect on their closest environment, such as their research group or institution.

All these processes are characterized by the fact that interconnections between groups tend to show a clear positive assortativity, widely observed in pairing from biological [105], sociological [106,107], and economic perspectives [108,109], as well as in import-export trade [110] and in the formation of international work teams as a result of globalization [111]. Furthermore, the four categories shown in Fig. 6 can be analyzed from a dynamic perspective in which social changes gradually produce modifications in the $\alpha$ and $\beta$ coefficients, leading to societies that become more permeable to intergroup connectivity with time [102].

\section{CONCLUSIONS}

In this paper, we introduce a theoretical framework describing the process by which individual decisions, based on a combination of self-interest and group-oriented behavior, drive the transition from small structures to robust and efficient, but also unequal, socioeconomic networks. One strength of our approach is that it can be used to study cases in which intra- and intercommunity links are qualitatively dissimilar and evolve at different rates, as happens in many real systems. Importantly, the present framework does not require that nodes in one network have prior information about the nodes of other networks. In consequence, it can be applied to scenarios in which nodes transiently explore new connections and abandon them if the result is not satisfactory.

Starting from two or more isolated communities, pairs of nodes from different communities can connect only if both nodes increase their centrality - or importance-after the connection. Likewise, old interactions are abandoned if the new ones are more beneficial. These two basic local assumptions are enough to produce a rich global process that first leads to a disordered connecting regime and finally reaches a hierarchical stationary state, defined by a Nash equilibrium, where the efficiency of the system - measured by the growth rate and the time to equilibrium of the processes that take place on it-is optimized.

The underdogs play a fundamental, but unrewarded role in the creation of these complex networked societies. In a context of competition among social or economic groups, sharing information, technology or wealth with other groups is detrimental for the strong networks as a whole; however, their weakest nodes receive such a little fraction of the total network centrality that becoming a critical connection between two groups overtakes the potential collective losses. While this process initially improves the status of the weak agents, the asymptotic establishment of an assortative connection pattern ends up pushing them back to peripheral positions. A parallel of this phenomenon has been pointed out by some recent studies, according to which social inequality might have its origin with the first farmers of Neolithic Europe [112], and only effective wealth-equalizing institutions might counter its otherwise inevitable growth in a globalized world [2].

Self-interest is not the only driving force that individuals follow in their interaction with other people and environments. However, when considering that payoffs are a linear combination of individual and collective interests, we still found that, in most cases, just a small amount of self-interest is enough for the system to evolve as if the group members were totally disengaged from the fate of their community. Therefore, only ensembles of small structures, sufficiently isolated from much larger networks and with very homogeneous topologies-as happens with small tribes or family businesses-where resources and wealth can be equally distributed might remain stable for long periods of time. Eventually, though, the growth of just one of these structures beyond a critical size can make the centrality of their nodes small enough to promote the search for self-benefit in some individuals, therefore launching the connecting process to the rest of small structures and destabilizing the system. This new perspective aligns with 
recent network-based explanations of the evolution and final extinction of primitive societies [112,113], as it happened during the transition from the politically egalitarian huntergatherers to the more complex and unequally distributed horticulturalist communities during the late Pleistocene and early Holocene.

One of the most fruitful approaches to explaining the emergence of social structures is based on the construction of cultural niches [114-116]. From an evolutionary point of view, the construction of niches is a process by which organisms modify their environment, thus altering the selection pressures on themselves and on other species. In the construction of cultural niches, social behaviors influence the evolution of other cultural or biological features, for example by modifying the transmissibility of certain cultural traits [117-119]. Our model can be easily framed in the context of cultural niche construction, regarding the integration of social groups through nonrandom pairing of their members. In particular, individual decisions on how to connect eventually modify the connecting behavior and overall centrality of the whole group. Assortative pairing, a ubiquitous property of human social networks, spontaneously emerges along the way. Assortative pairing is frequent not only in terms of partner choice $[120,121]$ but also in many other types of cultural interactions, called assortative meetings [122], and it has important implications as it facilitates the spread of rare cultural or genetic variants in the population $[117,123]$. Several mechanisms have been proposed to explain assortative pairing, such as phenotypic assortment based on mate choice $[124,125]$, partner interaction and convergence in phenotype over time [126,127], and homogamy according to social or environmental background [106,128,129]. In this context, our results suggest that assortative pairing could be, at least in part, an emergent property of the competitive mating process.

Beyond the context of pairing in religious, economic, social, or ethnic groups, the phenomenology described in this paper is compatible with the asymptotic topology of business affinity networks and friendship networks in rural communities, and with the evolution of scientific collaborations in an emergent area of research. Some other real networks whose evolution with time has already been studied in literature, such as the social contacts and mixing among students throughout elementary, middle, and high school in the context of the transmission of influenza [130], the school networks from the National Longitudinal Study of Adolescent Health (AddHealth) [131] or the 12-year (1988-1999) monitoring of the collaboration among institutions in the field of biotechnology [132], could become promising applications of this methodology. Furthermore, it would be of interest to test the effect of assigning a cost to (i) creating links - to account for the effort, time, and money invested in building new social or economic relationships - and (ii) rewiring links - to account for punishment to social climbers, a behavior that is frowned on in many social environments [133,134]. All in all, our framework highlights the relevance of eigenvector centrality for the analysis of socioeconomic networks and may inspire further studies about how social, technological, or economic networks interact to give rise to the complex structures that surround us.

\section{ACKNOWLEDGMENTS}

The authors acknowledge the assistance of D. Peralta-Salas in the analytical computations, fruitful conversations with $\mathrm{N}$. Aguirre, A. Alonso, S. Bowles, I. Briz Godino, P. Catalán, J. Cuesta, A. Delgado, L. Dudbridge, N. Fiorini, J.-M. Galán, O. Gómez, H. Gintis, S. Lozano, S. Manrubia, L. Prignano, F. Puente-Sánchez, A.-L. Sanz, and P. Yubero, and the support of the Spanish Ministerio de Ciencia, Innovación y Universidades under Project No. FIS2017-89773-P (MiMevo). J.A. is supported through Grant No. SEV 2017-0712 and the Spanish State Research Agency (AEI) Project No. MDM-2017-0737 Unidad de Excelencia María de Maeztu-Centro de Astrobiología (INTA-CSIC). J.I. is supported by the Ramón y Cajal Programme from the Spanish Ministry of Science (Grant No. RYC-2017-22524), the Agencia Estatal de Investigación of Spain (Grant No. PID2019-106618GA-I00), and the Severo Ochoa Programme for Centres of Excellence in R\&D from the Agencia Estatal de Investigación of Spain (Grant No. SEV2016-0672 (2017-2021) to the CBGP). F.P.-M. is supported through Grants No. CSO2016-74888-C4-4-R (CITITALENT, AEI/FEDER, UE), No. H2019/HUM-5761 (INNJOBMADCM) and 2019/00003/016/001/007 (COVID-19 UAH).

J.I. and J.A. conceived and designed the project, and performed the numerical simulations. J.A. developed the analytical work. J.I., F.P.-M. and J.A. wrote the paper and contributed to the interpretation of the results and their applicability to real data.

\section{APPENDIX A: BASIC DEFINITIONS AND ANALYTICAL TREATMENT OF THE PHENOMENOLOGY}

The eigenvector centrality gives a score to every node within a network that is proportional to the importance of its neighbors. The eigenvector centrality $x_{i}$ of node $i$ in network $\mathrm{G}$ follows

$$
x_{i}=\lambda_{1}^{-1} \sum_{j} G_{i j} x_{j},
$$

where $G_{i j}$ are the components of the adjacency matrix $\mathbf{G}$ and $\lambda_{1}$ its largest eigenvalue. The eigenvector centrality coincides with the eigenvector $\vec{u}_{1}$ of $\mathbf{G}$.

Let us focus on the simplest case of $m=2$ networks, A and B from now on, with $N_{A}$ and $N_{B}$ nodes, respectively, and $\lambda_{A, 1}>\lambda_{B, 1}$, such that $\mathrm{A}$ and $\mathrm{B}$ interconnect through a set $\{c l\}$ of $n$ connector links to give rise to a total interconnected network $\mathrm{T}$ of nodes $N_{T}=N_{A}+N_{B}$, largest eigenvalue $\lambda_{T, 1}$, and an associated eigenvector $\vec{u}_{T, 1}$ normalized such that $\sum_{k=1}^{N_{T}}\left(\vec{u}_{T}\right)_{k}^{2}=1$. The weights of connector links $\epsilon$ might be equal or different to those of the intralinks in A and B.

The centrality of each network as a whole is calculated accumulating that of all its nodes

$$
C_{\alpha}=\sum_{i \in \alpha} C_{i},
$$

where $C_{i}=\left(\vec{u}_{T}\right)_{i} /\left\|\vec{u}_{T}\right\|$ is the centrality of node $i$ in network $\alpha$. In the numerical simulations developed in this paper, $C_{i}$ is 
L1-normalized, that is,

$$
\left\|\vec{u}_{T}\right\|=\left\|\vec{u}_{T}\right\|_{1}=\sum_{k=1}^{N_{T}}\left(\vec{u}_{T}\right)_{k},
$$

and therefore $\sum_{k=1}^{N_{T}} C_{i}=1$. To simplify the analytical calculations of this Appendix, however, we will use an alternative definition for the centrality of a node where $C_{i}$ is L2normalized, that is,

$$
\left\|\vec{u}_{T}\right\|=\left\|\vec{u}_{T}\right\|_{2}=\sqrt{\sum_{k=1}^{N_{T}}\left(\vec{u}_{T}\right)_{k}^{2}}=1,
$$

and therefore $C_{i}=\left(\vec{u}_{T}\right)_{i}$ and $\sum_{k=1}^{N_{T}} C_{k}^{2}=1$. The results should be qualitatively equivalent, as proved in Ref. [37].

As shown in Ref. [76], the distribution of the centrality between two networks $\mathrm{A}$ and $\mathrm{B}$ that interconnect through a set $\{c l\}$ of connector links to give rise to an interconnected network $\mathrm{T}$ is extremely dependent on the connector nodes, as well as on the largest eigenvalues $\lambda_{A, 1}$ and $\lambda_{B, 1}$ of the isolated networks $\mathrm{A}$ and $\mathrm{B}$. The first eigenvalue and its associated eigenvector of $\mathrm{T}$, expressed as quantities that are only dependent on the isolated networks $\mathrm{A}$ and $\mathrm{B}$, can be approximated to second order by

$$
\begin{gathered}
\vec{u}_{T, 1}=\frac{\vec{u}_{A, 1}+\epsilon \sum_{j=1}^{N_{B}} a_{j} \vec{u}_{B, j}+\epsilon^{2} \sum_{i=2}^{N_{A}} \sum_{j=1}^{N_{B}} b_{i j} \vec{u}_{A, i}}{\sqrt{1+\epsilon^{2} \sum_{j=1}^{N_{B}} a_{j}^{2}}}+o\left(\epsilon^{3}\right), \\
\lambda_{T, 1}=\lambda_{A, 1}+\epsilon^{2} \sum_{j=1}^{N_{B}}\left(\vec{u}_{A, 1} \mathbf{P} \vec{u}_{B, j}\right) a_{j}+o\left(\epsilon^{3}\right),
\end{gathered}
$$

where $\quad a_{j}=\left(\vec{u}_{A, 1} \mathbf{P} \vec{u}_{B, j}\right) /\left(\lambda_{A, 1}-\lambda_{B, j}\right), \quad b_{i j}=a_{j}\left(\vec{u}_{A, i} \mathbf{P}\right.$ $\left.\vec{u}_{B, j}\right) /\left(\lambda_{A, 1}-\lambda_{A, i}\right), \epsilon$ is the strength (weight) of the connector links and $\mathbf{P}$ is a matrix formed by $P_{p q}=P_{q p}=1$ if nodes $p$ of $\mathrm{A}$ and $q$ of $\mathrm{B}$ are connected through a connector link, and $P_{p q}=P_{q p}=0$ elsewhere. $\vec{u}_{T, 1}, \vec{u}_{A, i}$, and $\vec{u}_{B, j}$ are vectors of L2-norm $\left\|\vec{u}_{T}\right\|_{2}=\left\|\vec{u}_{A, i}\right\|_{2}=\left\|\vec{u}_{B, j}\right\|_{2}=1$, and length $N_{A}+N_{B}$; the first $N_{A}$ elements of $\vec{u}_{A, i}$ are equal to the $i$ eigenvector of network $\mathrm{A}$ isolated, and the last $N_{B}$ elements of $\vec{u}_{B, j}$ are equal to the $j$ eigenvector of network B isolated. The rest are equal to zero.

Equation (A5) is very accurate to obtain the centrality of all nodes in two networks connected through a limited number of links, with the exception of the connector nodes, because they have an extra link with the opposite network that benefits them over the rest of the nodes of the network $[37,76]$. Combining Eqs. (A1), (A5), and (A6), we can approximate the centrality of a connector node $l$ in network A linked to node $m$ in B when both networks are interconnected through a set $\{c l\}$ of connector links by

$$
C_{\{c l\}, l \in A}=\left(\vec{u}_{T, 1}\right)_{l \in A}+\epsilon \frac{\left(\vec{u}_{T, 1}\right)_{m \in B}}{\lambda_{T, 1}}+o\left(\epsilon^{3}\right) .
$$

In a similar way, we can approximate the centrality of a connector node $m$ in network B linked to node $l$ in A when both networks are interconnected through a set $\{c l\}$ of connector links by

$$
C_{\{c l\}, m \in B}=\left(\vec{u}_{T, 1}\right)_{m \in B}+\epsilon \frac{\left(\vec{u}_{T, 1}\right)_{l \in A}}{\lambda_{T, 1}}+o\left(\epsilon^{3}\right) .
$$

In Eqs. (A7) and (A8), $\left(\vec{u}_{T, 1}\right)_{l}$ and $\lambda_{T, 1}$ are obtained from Eqs. (A5) and (A6).

If we neglect the $j>1$ elements in Eqs. (A5) and (A6) because they are far less relevant than the first element of the summations as $\lambda_{A, 1}-\lambda_{B, j}>\lambda_{A, 1}-\lambda_{B, 1}$ for $j>1$, and we neglect the term $\sum_{i=2}^{N_{A}} \sum_{j=1}^{N_{B}} b_{i j} \vec{u}_{A, i}$ because it only depends on the eigenvectors of $\mathrm{A}$ of order $i>1$ and are negligible in comparison to $\vec{u}_{A, 1}$, we obtain

$$
\begin{gathered}
C_{\{c l\}, l \in A} \sim\left[\left(\vec{u}_{A, 1}\right)_{l}+\frac{\epsilon^{2} a_{1, n}\left(\vec{u}_{B, 1}\right)_{m}}{\lambda_{A, 1}+\epsilon^{2} a_{1, n}\left(\vec{u}_{A, 1} \mathbf{P} \vec{u}_{B, 1}\right)}\right] / \sqrt{1+\epsilon^{2} a_{1, n}^{2}}, \\
C_{\{c l\}, m \in B} \sim \epsilon\left[a_{1, n}\left(\vec{u}_{B, 1}\right)_{m}+\frac{\left(\vec{u}_{A, 1}\right)_{l}}{\lambda_{A, 1}+\epsilon^{2} a_{1, n}\left(\vec{u}_{A, 1} \mathbf{P} \vec{u}_{B, 1}\right)}\right] / \sqrt{1+\epsilon^{2} a_{1, n}^{2}},
\end{gathered}
$$

where

$$
a_{1, n}=\frac{S_{n}}{\lambda_{A, 1}-\lambda_{B, 1}}
$$

and

$$
S_{n}=\vec{u}_{A, 1} \mathbf{P} \vec{u}_{B, 1}=\sum_{c l}\left(\vec{u}_{A, 1}\right)_{p} \cdot\left(\vec{u}_{B, 1}\right)_{q},
$$

being $p \in A, q \in B$ the $n$ pairs of nodes connected through connector links. Therefore, the strength of connections $S_{n}$ represents the strength of the union of both networks through $n$ links, as it is the sum of the products of the eigenvector centralities of all connector nodes measured when the networks are disconnected.

A development in powers of $\epsilon$ of Eqs. (A9) and (A10) helps to analyze the results and yields

(i) Centrality of node $l$ in A when there are $n$ connector links between networks $\mathrm{A}$ and $\mathrm{B}$ and $l$ is not connected to network B:

$$
C_{\{c l\}, l \in A} \sim\left(\vec{u}_{A, 1}\right)_{l}-\epsilon^{2} \frac{a_{1, n}^{2}\left(\vec{u}_{A, 1}\right)_{l}}{2} .
$$

(ii) Centrality of node $l$ in $\mathrm{A}$ when there are $n$ connector links between networks $\mathrm{A}$ and $\mathrm{B}$ and $l$ is connected to node $m$ in network B:

$$
C_{\{c l\} \cup\{l m\}, l \in A} \sim\left(\vec{u}_{A, 1}\right)_{l}+\epsilon^{2} \frac{a_{1, n}\left(\vec{u}_{B, 1}\right)_{m}}{\lambda_{A, 1}}-\epsilon^{2} \frac{a_{1, n}^{2}\left(\vec{u}_{A, 1}\right)_{l}}{2} .
$$

(iii) Centrality of node $m$ in $\mathrm{B}$ when there are $n$ connector links between networks $\mathrm{A}$ and $\mathrm{B}$ and $m$ is not connected to network A:

$$
C_{\{c l\}, m \in B} \sim \epsilon a_{1, n}\left(\vec{u}_{B, 1}\right)_{m} .
$$


(iv) Centrality of node $m$ in B when there are $n$ connector links between networks $\mathrm{A}$ and $\mathrm{B}$ and $m$ is connected to node $A$ in network A:

$$
C_{\{c l\} \cup\{l m\}, m \in B} \sim \epsilon\left[a_{1, n}\left(\vec{u}_{B, 1}\right)_{m}+\frac{\left(\vec{u}_{A, 1}\right)_{l}}{\lambda_{A, 1}}\right] .
$$

\section{Summary}

When a node from network A contacts a node from network $\mathrm{B}$, the new centrality of the node in A depends on the centrality it had in its original network isolated from the other and the addition of two quantities: one is always positive because it is the increase of its centrality due to a new connection [see Eq. (A1)] and the second is negative due to the change that its whole network suffers due to the addition of this new connection [see Eq. (A5) and Ref. [76], where it was shown that the addition of connector links between two isolated networks transmits centrality from the strong network to the weak one]. On the other hand, the payoff of the connector node in network B will always be positive when connecting to $\mathrm{A}$ because it increases due to the new connection and the transmission of centrality from A to B.

Finally, the payoff of a certain node $l$ of A or $m$ of B at time $t$ will then be $\Delta C=C(t)-C(t-1)$, being these expressions the corresponding from Eqs. (A11)-(A14). Only when node $l$ in $\mathrm{A}$ and $m$ in $\mathrm{B}$ receive a $\Delta C>0$ will the link $l m$ be accepted in the system.

\section{APPENDIX B: NUMERICAL TEST OF THE ANALYTICAL RESULTS}

Let us measure the quantitative precision of the analytic results presented above. We connect two scale-free networks of 50 nodes each by one single connector link in all possible configurations. For each connection between one node of network $\mathrm{A}$ and one of network B, we plot in Fig. 7 the connecting motivation for the connector node of network A, $\left(\vec{u}_{T, 1}\right)_{l} /\left(\vec{u}_{A, 1}\right)_{l}$. As this quantity represents the ratio between the centrality of the node obtained after connecting to network B and the centrality it had in network A isolated from B, only when it is larger than 1 will the new connector link be accepted. The result is calculated numerically in Fig. 7(a) and analytically in Fig. 7(b) following Eq. (A9). The connection boundaries $\left(\vec{u}_{T, 1}\right)_{l} /\left(\vec{u}_{A, 1}\right)_{l}=1$ are plotted in red. These boundaries show that only nodes in A of low centrality, i.e., peripheral nodes, accept to connect to network $\mathrm{B}$, while central nodes would prefer to remain isolated. The reason is that when two networks are connected through peripheral nodes, the system is weakly perturbed and only a low quantity of centrality is transferred from network A to B, as shown in Fig. 7(c). Therefore, this low loss can be balanced out by the connector node with the personal winnings due to obtaining a new connection. On the other hand, connecting through central nodes is highly detrimental for all nodes in the strong network, and therefore the extra centrality obtained by the hub for obtaining a new neighbor does not balance out its loss. Note that, in spite of the severe approximations done to obtain Eq. (A9), where instead of all the eigenspectra of A and B only the largest eigenvalues and their associated eigenvectors were used, the main properties of the phenomenology are recovered.
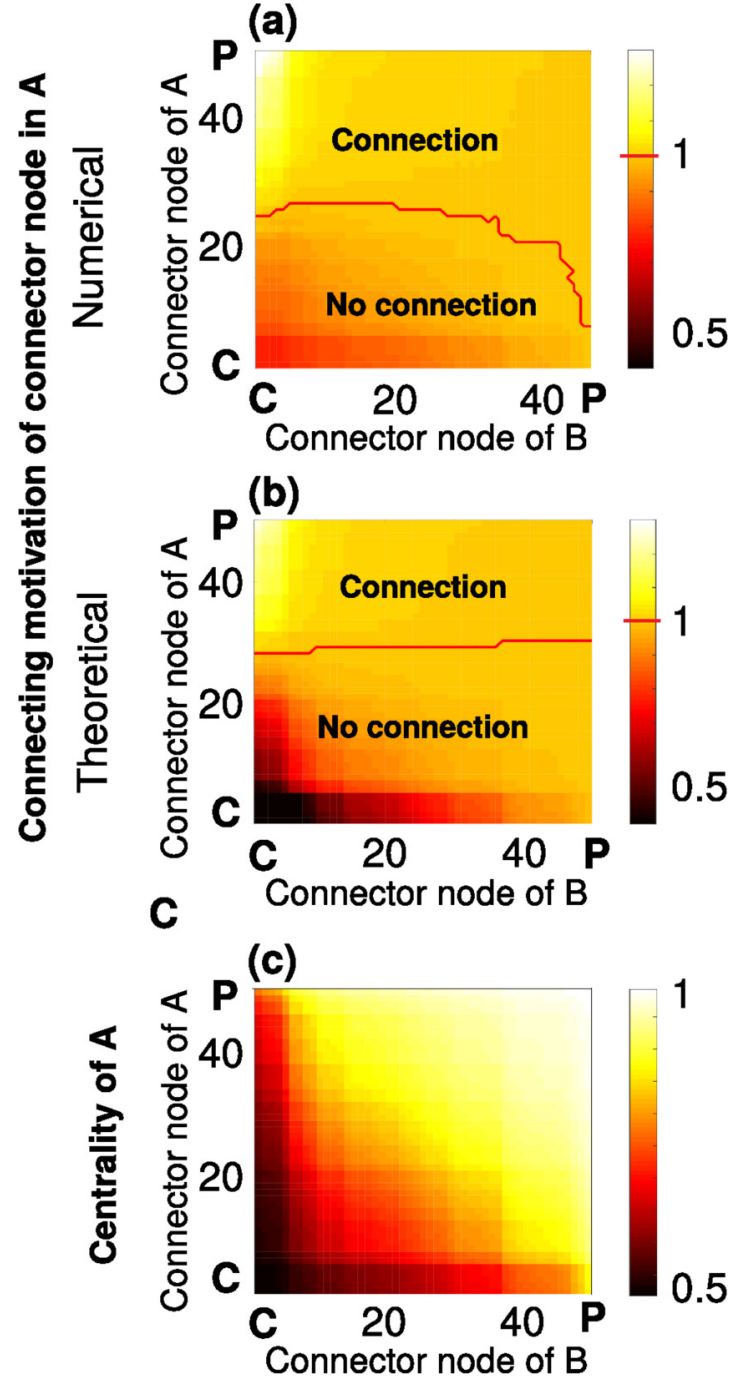

FIG. 7. Numerical test of the theoretical approach of the phenomenology. Two Barabási-Albert networks of 50 nodes each, $\lambda_{A, 1}=5.960$ and $\lambda_{B, 1}=5.952$, are connected through one single connector link of weight $\epsilon=0.5$ in all possible configurations $(50 \times$ 50). The axes represent the connector nodes in networks A and B, and nodes are numbered according to their network centrality rank. Central nodes $(\mathrm{C})$ and peripheral nodes $(\mathrm{P})$ are, respectively, those with higher and lower eigenvector centralities. (a), (b) show the connecting motivation of every node in strong network $\mathrm{A}$ when it is connected to a node in network B (numerically and analytically calculated, respectively). The connection boundaries (red curves) delimit the accepted connections. Only the peripheral nodes in A accept connecting to network B. In (c), the total centrality of network $\mathrm{A}, C_{A}$, is plotted.

\section{APPENDIX C: ANALYSIS OF THE THREE STAGES OF THE PROCESS}

\section{Stage 1: The approaching stage \\ The networks are disconnected}

Let us suppose the process is in its first step and therefore networks $\mathrm{A}$ and $\mathrm{B}$ are isolated, that is, $n=1$, the strength of connections $S_{n}=\vec{u}_{A, 1} \mathbf{P} \vec{u}_{B, 1}=\left(\vec{u}_{A, 1}\right)_{l} \cdot\left(\vec{u}_{B, 1}\right)_{m}$, and $S_{n-1}=0$. 
Network A. For node $l$ in A, we obtain from Eq. (A12)

$$
\Delta C_{l \in A}=\epsilon^{2}\left[\frac{a_{1, n}\left(\vec{u}_{B, 1}\right)_{m}}{\lambda_{A, 1}}-\frac{a_{1, n}^{2}\left(\vec{u}_{A, 1}\right)_{l}}{2}\right],
$$

where $a_{1, n}=S_{n} /\left(\lambda_{A, 1}-\lambda_{B, 1}\right)=\left(\vec{u}_{A, 1}\right)_{l}\left(\vec{u}_{B, 1}\right)_{m}$ because there is only one connector link.

Equalizing such payoff to zero yields the critical connecting centrality $\left(\vec{u}_{A, 1}\right)_{\text {crit }}$ for every node in network A: Only those with centrality (measured when network $\mathrm{A}$ is isolated from B) under that critical value will connect to network B. We obtain that

$$
\left(\vec{u}_{A, 1}\right)_{\text {crit }}=\sqrt{2\left(1-\frac{\lambda_{B, 1}}{\lambda_{A, 1}}\right)} .
$$

Network $B$. For node $m$ in network B, we obtain from Eq. (A14)

$$
\Delta C_{m \in B}=\epsilon\left[a_{1, n}\left(\vec{u}_{B, 1}\right)_{m}+\frac{\left(\vec{u}_{A, 1}\right)_{l}}{\lambda_{A, 1}}\right] .
$$

Therefore, $\Delta C_{m \in B}>0$ for all $\left(\vec{u}_{A, 1}\right)_{l}$ and $\left(\vec{u}_{B, 1}\right)_{m}$, that is, any node in $\mathrm{B}$ will accept the connection with a node in $\mathrm{A}$ when both networks are mutually disconnected because the initial centrality of all nodes in B is zero.

Summary. The first accepted connection between two networks A and B will necessarily go from any node of the weak network B to a peripheral node of the strong network A.

\section{First connections between networks $A$ and $B$}

Let us study the potential connection between nodes $l$ in A and $m$ in $\mathrm{B}$ when both networks are connected through a low number $n-1>0$ links, but nodes $l$ and $m$ are not connected with any node of the opposite network.

Network A. For node $l$ in A, we obtain from Eqs. (A11) and (A12)

$$
\Delta C_{l \in A}=\epsilon^{2}\left[\frac{a_{1, n}\left(\vec{u}_{B, 1}\right)_{m}}{\lambda_{A, 1}}-\frac{\left(\vec{u}_{A, 1}\right)_{l}}{2}\left(a_{1, n}^{2}-a_{1, n-1}^{2}\right)\right],
$$

where $a_{1, n}=S_{n} /\left(\lambda_{A, 1}-\lambda_{B, 1}\right), a_{1, n-1}=S_{n-1} /\left(\lambda_{A, 1}-\lambda_{B, 1}\right)$, and $S_{n}=S_{n-1}+\left(\vec{u}_{A, 1}\right)_{l}\left(\vec{u}_{B, 1}\right)_{m}$.

From here it is easy to obtain that node $l$ in A will accept the connection with node $m$ in B (i.e. $\Delta C_{l \in A}>0$ ) if

$$
F(x)=x^{3}+2 \mu x^{2}-2 c x-2 \mu c<0,
$$

where $x=\left(\vec{u}_{A, 1}\right)_{l}, \quad \mu=S_{n-1} /\left(\vec{u}_{B, 1}\right)_{m}, \quad$ and $c=\Delta \lambda / \lambda_{A, 1}$. Throughout the calculations $\Delta \lambda=\lambda_{A, 1}-\lambda_{B, 1}$.

We need to know the solutions of $F(x)=0$ to know the values of $\left(\vec{u}_{A, 1}\right)_{l}$ that verify $F(x)<0$. They can be obtained explicitly but are very complex expressions, so let us obtain as much information as possible about them and their relation to the parameters of the system. It is easy to verify that

(1) $F(0)=-2 c \mu<0$.

(2) $F^{\prime}(0)=-2 c<0$.

(3) $\lim _{x \rightarrow \infty} F(x)=\infty$.

(4) One root of $F^{\prime}(x)=3 x^{2}+2 \mu x-2 c=0$ is positive and one is negative.

All this yields that there is only one $x_{0}>0$ that verifies $F\left(x_{0}\right)=0$, and it verifies that $F(x) \leqslant 0$ for $x<x_{0}$ and $F^{\prime}\left(x_{0}\right)>0$. Therefore, that unique positive solution will be the critical connecting centrality $x_{0}=\left(\vec{u}_{A, 1}\right)_{\text {crit }}$ such that the nodes in A will connect to network B if $\left(\vec{u}_{A, 1}\right)_{l}<\left(\vec{u}_{A, 1}\right)_{\text {crit }}$. Let us analyze its dependency on $\mu$ and $c$ :

$$
\begin{aligned}
\frac{\partial F(x)}{\partial \mu} & =3 x_{0}^{2} \frac{\partial x_{0}}{\partial \mu}+2 x_{0}^{2}+4 \mu x_{0} \frac{\partial x_{0}}{\partial \mu}-2 c \frac{\partial x_{0}}{\partial \mu}-2 c=0 \\
& \Rightarrow \frac{\partial x_{0}}{\partial \mu}=\frac{2\left(c-x_{0}^{2}\right)}{3 x_{0}^{2}+4 \mu x_{0}-2 c} .
\end{aligned}
$$

Note that

$$
F^{\prime}\left(x_{0}\right)=3 x_{0}^{2}+4 \mu x_{0}-2 c \Rightarrow \frac{\partial x_{0}}{\partial \mu}=\frac{2\left(c-x_{0}^{2}\right)}{F^{\prime}\left(x_{0}\right)} .
$$

We have already shown that $F^{\prime}\left(x_{0}\right)>0$, therefore the sign of $\frac{\partial x_{0}}{\partial \mu}$ is that of $c-x_{0}^{2}$. From $F\left(x_{0}\right)=0$, we obtain that

$$
\begin{aligned}
c-x_{0}^{2} & =\frac{x_{0}\left(x_{0}^{2}-2 c\right)}{2 \mu} \\
\Rightarrow c-x_{0}^{2} & =\frac{-c x_{0} / 2 \mu}{1+x_{0} / 2 \mu} .
\end{aligned}
$$

As $c>0, \mu>0$, and $x_{0}>0$, we obtain that $c-x_{0}^{2}<0$ and therefore $\frac{\partial x_{0}}{\partial \mu}<0$.

In a similar way, we obtain for $c$ the following:

$$
\begin{aligned}
\frac{\partial F(x)}{\partial c} & =3 x_{0}^{2} \frac{\partial x_{0}}{\partial c}+4 \mu x_{0} \frac{\partial x_{0}}{\partial c}-2 x_{0}-2 c \frac{\partial x_{0}}{\partial c}-2 \mu=0 \\
& \Rightarrow \frac{\partial x_{0}}{\partial c}=\frac{2\left(x_{0}+\mu\right)}{3 x_{0}^{2}+4 \mu x_{0}-2 c}=\frac{2\left(x_{0}+\mu\right)}{F^{\prime}\left(x_{0}\right)} .
\end{aligned}
$$

As $x_{0}+\mu>0$ and $F^{\prime}\left(x_{0}\right)$, we obtain that $\frac{\partial x_{0}}{\partial c}>0$.

In summary, as $x=\left(\vec{u}_{A, 1}\right)_{\text {crit }}, \mu=S_{n-1} /\left(\vec{u}_{B, 1}\right)_{m}$, and $c=$ $\Delta \lambda / \lambda_{A, 1}$, we finally obtain that $\frac{\partial\left(\vec{u}_{A, 1}\right)_{\text {crit }}}{\partial S_{n}}<0, \frac{\partial\left(\vec{u}_{A, 1}\right)_{\text {crit }}}{\partial\left(\vec{u}_{B, 1}\right)_{m}}>0$ and $\frac{\partial\left(\vec{u}_{A, 1}\right)_{\text {crit }}}{\partial\left(\lambda_{B, 1} / \lambda_{A, 1}\right)}<0$.

Network B. For node $m$ in network B, we obtain from Eqs. (A13) and (A14)

$$
\begin{aligned}
\Delta C_{m \in B} & =\epsilon\left[a_{1, n}\left(\vec{u}_{B, 1}\right)_{m}+\frac{\left(\vec{u}_{A, 1}\right)_{l}}{\lambda_{A, 1}}\right]-\epsilon a_{1, n-1}\left(\vec{u}_{B, 1}\right)_{m} \\
& =\epsilon\left[\frac{\left(\vec{u}_{B, 1}\right)_{m}^{2}}{\Delta \lambda}+\frac{1}{\lambda_{A, 1}}\right],
\end{aligned}
$$

where $a_{1, n}=S_{n} /\left(\lambda_{A, 1}-\lambda_{B, 1}\right), a_{1, n-1}=S_{n-1} /\left(\lambda_{A, 1}-\lambda_{B, 1}\right)$, and $S_{n}=S_{n-1}+\left(\vec{u}_{A, 1}\right)_{l}\left(\vec{u}_{B, 1}\right)_{m}$. Therefore, $\Delta C_{m \in B}>0$ for all nodes $m$ in $\mathrm{B}$ that connect to any node of $\mathrm{A}$ when there are $n-1$ links between $\mathrm{A}$ and $\mathrm{B}$.

Summary. The more similar the eigenvalues $\lambda_{A, 1}$ and $\lambda_{B, 1}$ of both networks, the more and more central connector links existing between them $\left(S_{n-1}\right)$ and the lower the centrality of node $m$ in $\mathrm{B}$, the weaker must be the connector node in network A to accept the connection.

Note that the critical value for the centrality of node $l$ in $\mathrm{A}$ is $\left(\vec{u}_{A, 1}\right)_{\text {crit }}=\sqrt{\frac{2 \Delta \lambda}{\lambda_{A, 1}}}$ for the first connector link and then decreases toward $\sqrt{\frac{\Delta \lambda}{\lambda_{A, 1}}}$ when $S_{n} \rightarrow \infty$. That is, the first connector links will hinder the connection from A to B and will only enable the connection of the most peripheral nodes in A to any node in B. As a consequence, the system will be dissortative during the first steps of the process. 


\section{Stage 2: The connecting stage}

As mentioned above, the approaching stage leads to networks $\mathrm{A}$ and $\mathrm{B}$ connected through a limited number of links that connect nodes of any centrality in B with the peripheral nodes in A. Let us show here how the nonperipheral nodes in A enter the game. To do this, we will study the potential connection between a node $l$ in A that is not connected with any node of $\mathrm{B}$, and a node $m$ in $\mathrm{B}$ such that is already connected with a node $l^{\prime}$ of $\mathrm{A}$ and faces breaking this link to connect to $l$. Note that $\mathrm{A}$ and $\mathrm{B}$ are connected through $n$ links before and after the rewiring.

\section{a. Network $A$}

For node $l$ in A, we obtain from Eqs. (A11) and (A12)

$$
\Delta C_{l \in A}=\epsilon^{2}\left[\frac{a_{1, n}\left(\vec{u}_{B, 1}\right)_{m}}{\lambda_{A, 1}}-\frac{\left(\vec{u}_{A, 1}\right)_{l}}{2}\left(a_{1, n}^{2}-a_{1, n^{\prime}}^{2}\right)\right],
$$

where $a_{1, n}=S_{n} /\left(\lambda_{A, 1}-\lambda_{B, 1}\right), a_{1, n^{\prime}}=S_{n^{\prime}} /\left(\lambda_{A, 1}-\lambda_{B, 1}\right)$, and $S_{n}=S_{n^{\prime}}+\left(\vec{u}_{A, 1}\right)_{l}\left(\vec{u}_{B, 1}\right)_{m}-\left(\vec{u}_{A, 1}\right)_{l^{\prime}}\left(\vec{u}_{B, 1}\right)_{m} . l^{\prime}$ is the node of A that is initially connected to $m, n^{\prime}$ refers to the links before the rewiring and $n$ to the links after the rewiring. (Note that the connector links after the rewiring are the same as before but where the connector link that connected $l^{\prime}$ and $m$ has been replaced by the connection between $l$ and $m$ ).

Neglecting terms of fourth order on the centrality terms, we approximate

$$
S_{n}^{2}-S_{n^{\prime}}^{2} \sim 2 S_{n^{\prime}}\left(\vec{u}_{B, 1}\right)_{m}\left[\left(\vec{u}_{A, 1}\right)_{l}-\left(\vec{u}_{A, 1}\right)_{l^{\prime}}\right] .
$$

From here, it is easy to obtain that node $l$ in A will accept the connection with node $m$ in $\mathrm{B}$ (i.e., $\Delta C_{l \in A}>0$ ) if

$$
\begin{aligned}
& \left(\vec{u}_{A, 1}\right)_{l}<\left(\vec{u}_{A, 1}\right)_{\text {crit }} \\
& \quad=\frac{\left(\vec{u}_{A, 1}\right)_{l^{\prime}}}{2}+\frac{c}{2 \mu}+\sqrt{\left[\frac{\left(\vec{u}_{A, 1}\right)_{l^{\prime}}}{2}+\frac{c}{2 \mu}\right]^{2}+c\left[1-\frac{\left(\vec{u}_{A, 1}\right)_{l^{\prime}}}{\mu}\right]} .
\end{aligned}
$$

Regarding $c=\Delta \lambda / \lambda_{A, 1}>0$ and $\mu=S_{n^{\prime}} /\left(\vec{u}_{B, 1}\right)_{m}>0$, it is clear that $\left(\vec{u}_{A, 1}\right)_{\text {crit }}-\left(\vec{u}_{A, 1}\right)_{l^{\prime}}>0$ if $\left(\vec{u}_{A, 1}\right)_{l^{\prime}}<\mu$, and this condition is verified for all $\left(\vec{u}_{A, 1}\right)_{l^{\prime}}$ because $\left(\vec{u}_{A, 1}\right)_{l} \cdot\left(\vec{u}_{B, 1}\right)_{m}<S_{n^{\prime}}$ when networks $\mathrm{A}$ and $\mathrm{B}$ are connected through $n>1$ link. Therefore, node $l$ in $\mathrm{A}$ will accept to connect to a node $m$ in B already connected to a node $l^{\prime}$ of centrality lower than that of $l$ (that is, when $\left.\Delta \vec{u}_{A, 1}=\left(\vec{u}_{A, 1}\right)_{l}-\left(\vec{u}_{A, 1}\right)_{l^{\prime}}>0\right)$ if $\left(\vec{u}_{A, 1}\right)_{l^{\prime}}<\left(\vec{u}_{A, 1}\right)_{l}<\left(\vec{u}_{A, 1}\right)_{\text {crit }}$.

Let us analyze the dependency of $\left(\vec{u}_{A, 1}\right)_{\text {crit }}$ on $S_{n^{\prime}}$ :

$$
\frac{\partial\left(\vec{u}_{A, 1}\right)_{\text {crit }}}{\partial \mu}=-\frac{c}{2 \mu}\left(1+\frac{K}{\sqrt{K^{2}+c}}\right),
$$

where $K=\frac{\left(\vec{u}_{A, 1}\right)_{l^{\prime}}}{2}-\frac{c}{2 \mu}$. Independently of the sign and value of $K$, it is easy to see that $\left|\frac{K}{\sqrt{K^{2}+c}}\right|<1$, and therefore $\frac{\partial\left(\vec{u}_{A, 1}\right)_{\text {crit }}}{\partial \mu}<0$. In summary, as $\mu=S_{n^{\prime}} /\left(\vec{u}_{B, 1}\right)_{m}, \frac{\partial\left(\vec{u}_{A, 1}\right)_{\text {crit }}}{\partial S_{n^{\prime}}}<0$ for all values of the parameters, and $\left(\vec{u}_{A, 1}\right)_{\text {crit }} \rightarrow \frac{\left(\vec{u}_{A, 1}\right)_{l^{\prime}}}{2}+$ $\sqrt{\frac{\Delta \lambda}{\lambda_{A, 1}}+\left(\frac{\left(\vec{u}_{A, 1}\right)_{l^{\prime}}}{2}\right)^{2}}$ when $S_{n^{\prime}} \rightarrow \infty$.
Regarding the dependency of $\left(\vec{u}_{A, 1}\right)_{\text {crit }}$ on $\left(\vec{u}_{A, 1}\right)_{l^{\prime}}$, we obtain

$$
\frac{\partial\left(\vec{u}_{A, 1}\right)_{\text {crit }}}{\partial\left(\vec{u}_{A, 1}\right)_{l^{\prime}}}=\frac{1}{2}\left(1+\frac{K}{\sqrt{K^{2}+c}}\right) .
$$

Once again, $\left|\frac{K}{\sqrt{K^{2}+c}}\right|<1$ for all $K$. As a consequence, $\frac{\partial\left(\vec{u}_{A, 1}\right)_{\text {crit }}}{\partial\left(u_{A, 1}\right)_{l^{\prime}}}>0$ for all values of the parameters, and $\left(\vec{u}_{A, 1}\right)_{\text {crit }} \rightarrow$ $\left(\vec{u}_{A, 1}\right)_{l^{\prime}}$ when $\left(\vec{u}_{A, 1}\right)_{l^{\prime}} \rightarrow \infty$.

\section{b. Network B}

For node $m$ in B, we obtain from Eqs. (A13) and (A14)

$$
\begin{aligned}
\Delta C_{m \in B} & =\epsilon\left[\left(\vec{u}_{B, 1}\right)_{m}\left(a_{1, n}-a_{1, n^{\prime}}\right)+\frac{\Delta \vec{u}_{A, 1}}{\lambda_{A, 1}}\right] \\
& =\epsilon \Delta \vec{u}_{A, 1}\left[\frac{\left(\vec{u}_{B, 1}\right)_{m}^{2}}{\Delta \lambda}+\frac{1}{\lambda_{A, 1}}\right] .
\end{aligned}
$$

It is clear that $\Delta C_{m \in B}>0$ if $\Delta \vec{u}_{A, 1}>0$, that is, node $m$ in $\mathrm{B}$ will only rewire its connection from $l^{\prime}$ to $l$ in $\mathrm{A}$ if its new neighbor $l$ has a larger centrality (measured in A isolated) than the old one, that is, if $\Delta \vec{u}_{A, 1}>0$.

\section{Summary}

The nodes $l$ in A that are not connected to B and the nodes $m$ in B that are already connected to nodes $l^{\prime}$ in A will connect through a new connector link as far as $\left(\vec{u}_{A, 1}\right)_{l^{\prime}}<\left(\vec{u}_{A, 1}\right)_{l}<$ $\left(\vec{u}_{A, 1}\right)_{\text {crit }}$, where $\left(\vec{u}_{A, 1}\right)_{\text {crit }}$ is given by Eq. (C8). When the number of connector links grows sufficiently (i.e., $S_{n^{\prime}}$ grows), this inequality becomes

$$
\left(\vec{u}_{A, 1}\right)_{l^{\prime}}<\left(\vec{u}_{A, 1}\right)_{l}<\frac{\left(\vec{u}_{A, 1}\right)_{l^{\prime}}}{2}+\sqrt{\frac{\Delta \lambda}{\lambda_{A, 1}}+\left[\frac{\left(\vec{u}_{A, 1}\right)_{l^{\prime}}}{2}\right]^{2}} .
$$

This result yields that at the beginning of the connecting stage, nodes in B connecting peripheral nodes in A will tend to change their connector links to nodes with a centrality slightly larger than their former connections. The critical centrality $\left(\vec{u}_{A, 1}\right)_{\text {crit }}$ now increases with $\left(\vec{u}_{A, 1}\right)_{l^{\prime}}$, which means that every new rewiring increases the value of the critical centrality, and leads to a cascade of new rewirings that ends with the majority of nodes in A and B interconnected in a disordered manner of null assortativity (see Sec. IV A of the main text for more details).

\section{Stage 3: The optimization stage}

At the beginning of this regime, networks $\mathrm{A}$ and $\mathrm{B}$ are already disorderly connected through a large number $n$ of connector links. Let us show here how these connector links self-organize as an emergent process to yield a new total network that is optimal in dynamical properties such as the growth rate or the time to equilibrium. To do this, we will study the potential connection between a node $l$ in A that is already connected to a node $m^{\prime}$, and a node $m$ in $\mathrm{B}$ that that is already connected with a node $l^{\prime}$ of $\mathrm{A}$. This leads to

$$
S_{n}=S_{n^{\prime}}+\left(\vec{u}_{A, 1}\right)_{l}\left(\vec{u}_{B, 1}\right)_{m}-\left(\vec{u}_{A, 1}\right)_{l^{\prime}}\left(\vec{u}_{B, 1}\right)_{m}-\left(\vec{u}_{A, 1}\right)_{l}\left(\vec{u}_{B, 1}\right)_{m^{\prime}} .
$$

Note that $n^{\prime}$ refers to the $n$ links before the new connection and $n$ to the $n-1$ links after the new connection and the deletion 
of two old links, but as the number of connector links $n$ is now of the order of the size of the smallest network, we can approximate $S_{n} \sim S_{n^{\prime}}$.

\section{a. Network A}

For a node $l$ in A already connected to a node $m^{\prime}$ in B that changes its link toward node $m$, we obtain from Eq. (A12)

$$
\Delta C_{l \in A}=\epsilon^{2} \frac{a_{1, n} \Delta \vec{u}_{B, 1}}{\lambda_{A, 1}}=\epsilon^{2} \frac{S_{n} \Delta \vec{u}_{B, 1}}{\lambda_{A, 1} \Delta \lambda},
$$

where $\Delta \vec{u}_{B, 1}=\left(\vec{u}_{B, 1}\right)_{m}-\left(\vec{u}_{B, 1}\right)_{m^{\prime}}$. It is clear that $\Delta C_{l \in A}>0$ if $\Delta \vec{u}_{B, 1}>0$.

\section{b. Network B}

For a node $m$ in B already connected to a node $l^{\prime}$ in A that changes its link toward node $l$, we obtain from Eq. (A14)

$$
\Delta C_{m \in B}=\epsilon \frac{\Delta \vec{u}_{A, 1}}{\lambda_{A, 1}},
$$

where $\Delta \vec{u}_{A, 1}=\left(\vec{u}_{A, 1}\right)_{l}-\left(\vec{u}_{A, 1}\right)_{l^{\prime}}$. It is clear that $\Delta C_{m \in B}>0$ if $\Delta \vec{u}_{A, 1}>0$.

\section{Summary}

During the optimization stage any node in A or B will accept a new connection to the opposite network as far as the centrality of the new neighbor is larger than that of the old one. This systematic acceptance of more central connections and rejection of more peripheral ones by the nodes of both networks yields to a systematic ordering of the connector links such that, after an intensive rewiring process, nodes in A become connected to nodes in B of the same position in the ranking of their network centralities (i.e., the most central node in A will become connected to the most central node in $\mathrm{B}$, the second to the second, and successively, and only the most peripheral nodes will remain disconnected to the opposite network). In consequence, the assortativity grows to its maximum positive value at the end of this stage.

A Nash equilibrium is the solution of a noncooperative game involving two or more players, in which each player is assumed to know the equilibrium strategies of the other players, and no player has anything to gain by changing only their own strategy. According to this definition, it is straightforward to see that the final configuration achieved by the system is a Nash equilibrium, as once all connector links are ordered according to the centrality of both networks, it will be impossible to find a rewiring that enables the simultaneous improvement of the centrality of both nodes.

\section{APPENDIX D: THE EQUILIBRIUM STATE OPTIMIZES THE DYNAMICAL PROPERTIES OF THE SYSTEM}

Let us prove in this Appendix that the Nash equilibrium reached at the end of the optimization stage optimizes both the growth rate and the time to equilibrium of the potential processes that could take place on the system.

The first and second eigenvalues associated to two networks $\mathrm{A}$ and $\mathrm{B}$ that connect through a set $\{c l\}$ of connector links to give rise to an interconnected network T, expressed as quantities that are only dependent on the isolated networks A and B, can be approximated to second order by (see Ref. [76])

$$
\begin{aligned}
& \lambda_{T, 1}=\lambda_{A, 1}+\epsilon^{2} \frac{\left(\vec{u}_{A, 1} \mathbf{P} \vec{u}_{B, 1}\right)^{2}}{\lambda_{A, 1}-\lambda_{B, 1}}+O\left(\epsilon^{2}\right), \\
& \lambda_{T, 2}=\lambda_{B, 1}-\epsilon^{2} \frac{\left(\vec{u}_{A, 1} \mathbf{P} \vec{u}_{B, 1}\right)^{2}}{\lambda_{A, 1}-\lambda_{B, 1}}+O\left(\epsilon^{2}\right) .
\end{aligned}
$$

As the growth rate of the process is given by $\lambda_{T, 1}$ and the time to equilibrium $t_{\mathrm{eq}}$ is proportional to $\left[\ln \left(\lambda_{T, 1} / \lambda_{T, 2}\right)\right]^{-1}$, it is clear that $\lambda_{T, 1}$ will be maximum and $t_{\mathrm{eq}}$ minimum when $\vec{u}_{A, 1} \mathbf{P} \vec{u}_{B, 1}$ is maximum. We will show that this happens at the Nash equilibrium.

First, let us recall that the strength of connections $S_{n}$ verifies

$$
S_{n}=\vec{u}_{A, 1} \mathbf{P} \vec{u}_{B, 1}=\sum_{c l}\left(\vec{u}_{A, 1}\right)_{p} \cdot\left(\vec{u}_{B, 1}\right)_{q},
$$

being $p \in A, q \in B$ the $n$ pairs of nodes connected through connector links. That is, $S_{n}$ is the sum of the products of the eigenvector centralities of all connector nodes measured when the networks are disconnected.

Let us define vector $\vec{x}$ such that $x_{i}=\left(\vec{u}_{A, 1}\right)_{i}$ if node $i$ in $\mathrm{A}$ is connected to $\mathrm{B}$ and $x_{i}=0$ otherwise, and vector $\vec{y}$ such that $y_{i}=\left(\vec{u}_{B, 1}\right)_{i}$ if node $i$ in $\mathrm{B}$ is connected to $\mathrm{A}$ and $x_{i}=0$ otherwise. Without any loss of generality let us suppose that $\vec{x}$ is sorted to verify $x_{i} \geqslant x_{i+1}$ and $\vec{y}$ is sorted in a way that node $i$ in $\mathrm{A}$ is connected to node $i$ for $i \leqslant n$. Note that vectors $\vec{x}$ and $\vec{y}$ have $n$ positive elements because there are $n$ connector links between A and B. According to these definitions, $S_{n}=$ $\vec{u}_{A, 1} \mathbf{P} \vec{u}_{B, 1}=\vec{x} \cdot \vec{y}$.

Our target is to find the sorting of $\vec{x}$ and $\vec{y}$ (that is, the configuration of connector links between $\mathrm{A}$ and $\mathrm{B}$ ) that maximizes $S_{n}$. As there are $\left(N_{A}+N_{B}\right)$ ! different permutations of the elements of $\vec{y}$, there are $\left(N_{A}+N_{B}\right)$ ! different configurations. If we start with a generic configuration 1 and permute two connections (i.e., two nodes $j$ and $k$ such that $k>j$ of one network swap their connector links to the other network) to reach a new configuration 2 , the change in the quantity $S_{n}$ is

$$
\Delta S_{n}=\left(S_{n}\right)_{2}-\left(S_{n}\right)_{1}=-\left(x_{j}-x_{k}\right)\left(y_{j}-y_{k}\right)=-\Delta x \Delta y .
$$

Regarding Eq. (D3), it is straightforward that the only configuration that verifies $\Delta S_{n}<0$ for all values of $j$ and $k>j$ is the ordered configuration such that $x_{i} \geqslant x_{i+1}$ and $y_{i} \geqslant y_{i+1} \forall i$, and therefore this configuration is a local maximum. Furthermore, any other configuration has at least two crossing connector links - that is, that verify $\Delta x \Delta y<0$ such that, when they are swapped, $\Delta S_{n}>0$. In consequence, all configurations with the exception of the ordered one are not local maxima and can increase their value of $S_{n}$ uncrossing one by one all their crossed connector links, until they reach the ordered configuration and maximize $S_{n}$.

In summary, $S_{n}=\vec{u}_{A, 1} \mathbf{P} \vec{u}_{B, 1}$ reaches a global maximum when the matrix $\mathbf{P}$ connects the nodes of $\mathrm{A}$ and $\mathrm{B}$ that share the same position in the ranking of centrality in $\vec{u}_{A, 1}$ and $\vec{u}_{B, 1}$. This yields that $\lambda_{T, 1}$ will be maximum and $t_{\mathrm{eq}}$ minimum when the most central nodes of $\mathrm{A}$ and $\mathrm{B}$ are interconnected, the second most central too, and successively. 


\section{APPENDIX E: WHEN INDIVIDUAL AND COLLECTIVE INTERESTS COMPETE}

In many social and economic networks, the individuals make decisions based on both group and individual motivations. To study this situation, here we analyze what happens if the centrality of an individual is redefined as a mixture of individual and collective interests. Following Ref. [84], we propose

$$
C_{\{c l\}, l \in A}=\alpha \frac{\left(\vec{u}_{T, 1}\right)_{l \in A}}{\left\|\vec{u}_{T, 1}\right\|}+(1-\alpha) \frac{\sum_{i \in A, i \neq l}\left(\vec{u}_{T, 1}\right)_{i}}{\left(N_{A}-1\right)\left\|\vec{u}_{T, 1}\right\|},
$$

where $C_{\{c l\}, l \in A}$ is the centrality of node $l$ of $\mathrm{A}$ when $\mathrm{A}$ and $\mathrm{B}$ are connected through the set $\{c l\}$ of $n$ connector links to form the total network T, and $\vec{u}_{T, 1}$ is the eigenvector associated to the maximum eigenvalue of the adjacency matrix of T. $\alpha$ is the self-interest parameter, and Eq. (E1) represents Eq. (2) where the normalized social reach $\beta=1 . \alpha=1$ is the situation in which the payoff is that of the connector node individually - the case studied above-and $\alpha=0$ is the case in which the payoff is that of the whole network A (excluding the connector node). Note that, while in the former Appendices the centralities were L2-normalized for simplicity (see Appendix A for details), in this Appendix we will make use of the same normalization used in the numerical simulations [L1-norm, see Eq. (A3)]. While it is more troublesome because it includes the term $\|\vec{u}\|_{1}=\sum_{i} u_{i}$ in the equations, we do it because the L1-norm of vectors is deeply related to the topology of the networks, and we will see that such topology strongly influences the phenomenology.

If networks $\mathrm{A}$ and $\mathrm{B}$ were initially disconnected, and then they connect through a single connector link that joins node $l$ in A with node $m$ in $\mathrm{B}$, the payoff obtained by node $l$ in $\mathrm{A}$ is

$$
\Delta C_{l \in A}=C_{\{c l\} \cup\{l m\}, l \in A}-C_{\{c l\}, l \in A},
$$

where

$$
C_{\{c l\}, l \in A}=\alpha \frac{\left(\vec{u}_{A, 1}\right)_{l}}{\left\|\vec{u}_{A, 1}\right\|_{1}}+(1-\alpha) \frac{\sum_{i \in A, i \neq l}\left(\vec{u}_{A, 1}\right)_{i}}{\left(N_{A}-1\right)\left\|\vec{u}_{A, 1}\right\|_{1}}
$$

is the centrality of node $l$ in A when networks A and B are still disconnected, and

$$
C_{\{c l\} \cup\{l m\}, l \in A}=\alpha\left(\vec{u}_{T, 1}\right)_{l, l}+(1-\alpha) \frac{\sum_{i \in A, i \neq l}\left(\vec{u}_{T, 1}\right)_{i, l}}{N_{A}-1}
$$

is the centrality of node $l$ in A after its connection with node $m$ in $\mathrm{B}$ (the second subindex of $\left(u_{T, 1}\right)$ remarks that the networks are connected through node $l$ in A). Note that $\left\|\vec{u}_{T, 1}\right\|_{1}=1$.

Following Eq. (A9), we obtain

$$
\begin{gathered}
\left(\vec{u}_{T, 1}\right)_{l, l} \sim \frac{\left(\vec{u}_{A, 1}\right)_{l}+\frac{\epsilon^{2} a_{1, n}\left(\vec{u}_{B, 1}\right)_{m}}{\lambda_{A, 1}+\epsilon^{2} a_{1, n}\left(\vec{u}_{A, 1} \mathbf{P} \vec{u}_{B, 1}\right)}}{\left\|\vec{u}_{A, 1}\right\|_{1}+\epsilon a_{1, n}\left\|\vec{u}_{B, 1}\right\|_{1}}, \\
\left(\vec{u}_{T, 1}\right)_{i \neq l, l} \sim \frac{\left(\vec{u}_{A, 1}\right)_{l}}{\left\|\vec{u}_{A, 1}\right\|_{1}+\epsilon a_{1, n}\left\|\vec{u}_{B, 1}\right\|_{1}},
\end{gathered}
$$

where $a_{1, n}=\left(\vec{u}_{A, 1} \mathbf{P} \vec{u}_{B, 1}\right) /\left(\lambda_{A, 1}-\lambda_{B, 1}\right)$ and $\vec{u}_{A, 1} \mathbf{P} \vec{u}_{B, 1}=$ $\left(\vec{u}_{A, 1}\right)_{l}\left(\vec{u}_{A, 1}\right)_{m}$ because there is only one connector link, lm.

If we introduce Eqs. (E5) and (E6) in Eq. (E4), and Eqs. (E3) and (E4) in Eq. (E2), we obtain an analytical expression for the payoff $\Delta C$. Developing $\Delta C$ in series of $\epsilon$, and equalizing it to zero, we finally obtain the critical value of $\alpha_{\text {crit }}$ such that the connection between both networks is possible if and only if $\alpha>\alpha_{\text {crit }}$,

$$
\alpha_{\text {crit }} \sim \frac{\left\|\vec{u}_{B, 1}\right\|_{1}\left(\left\|\vec{u}_{A, 1}\right\|_{1}-\left(u_{A, 1}\right)_{l}\right) M_{1}}{\left\|\vec{u}_{B, 1}\right\|_{1} M_{1} M_{2}+\epsilon\left\|\vec{u}_{A, 1}\right\|_{1} \Delta \lambda\left(N_{A}-1\right)\left(u_{B, 1}\right)_{m}},
$$

where

$$
\begin{aligned}
& M_{1}=\lambda_{A, 1} \Delta \lambda+\epsilon^{2}\left(u_{A, 1}\right)_{l}^{2}\left(u_{B, 1}\right)_{m}^{2}, \\
& M_{2}=\left\|\vec{u}_{A, 1}\right\|_{1}-N_{A}\left(u_{A, 1}\right)_{l} .
\end{aligned}
$$

\section{Dependency of the critical self-interest parameter $\alpha_{\text {crit }}$ on the main parameters of the system}

The complexity of the explicit solution for $\alpha_{\text {crit }}$ makes the analysis of its dependency on the different parameters of the system a nontrivial task. Note, in addition, that in most types of networks varying one parameter implies varying some of the rest.

\section{a. Dependency on the maximum eigenvalues}

The analysis of $\frac{\partial \alpha_{\text {crit }}}{\partial \lambda_{A, 1}}$ implies that $\alpha_{\text {crit }}$ grows with $\lambda_{A, 1}$ for $\lambda_{A, 1}>\lambda_{B, 1}+\epsilon\left(u_{A, 1}\right)_{l}\left(u_{B, 1}\right)_{m}$, and $\alpha \rightarrow \frac{\left\|\vec{u}_{A, 1}\right\|_{1}-\left(u_{A, 1}\right)_{l}}{\left\|\vec{u}_{A, 1}\right\|_{1}-N_{A}\left(u_{A, 1}\right)_{l}}>$ 1 when $\lambda_{A, 1} \rightarrow \infty$. On the other hand, $\frac{\partial \alpha_{\text {crit }}}{\partial \lambda_{B, 1}}$ implies that $\alpha_{\text {crit }}$ is approximately constant for low values of $\lambda_{B, 1}$ and $\alpha \rightarrow \infty$ in $\lambda_{B, 1}=\lambda_{A, 1}+L$, where

$$
L=\frac{\epsilon^{2}\left\|\vec{u}_{B, 1}\right\|_{1} M_{2}\left(u_{A, 1}\right)_{l}^{2}\left(u_{B, 1}\right)_{m}^{2}}{\lambda_{A, 1}\left\|\vec{u}_{B, 1}\right\|_{1} M_{2}+\epsilon\left(N_{A}-1\right)\left(u_{A, 1}\right)_{l}\left(u_{B, 1}\right)_{m}} .
$$

In summary, $\alpha_{\text {crit }}$ grows with $\lambda_{A, 1}$ (toward a maximum bounded value) and with $\lambda_{B, 1}$ (toward infinity for a finite value of $\left.\lambda_{B, 1}\right)$. This is in qualitative agreement with the numerical results shown in Figs. 3(e) and 3(g).

\section{b. Dependency on the centrality of the nodes of the strong network}

Let us study the dependency of $\alpha_{\text {crit }}$ on the normalized centrality of the nodes of the strong network $\left(u_{A, 1}\right)_{l} /\left\|\vec{u}_{A, 1}\right\|_{1}$. If we neglect the terms in Eq. (E7) that are proportional to $\epsilon^{2}\left(\left(u_{A, 1}\right)_{l}\right)^{2}\left(\left(u_{B, 1}\right)_{m}\right)^{2}$ because they are much smaller than the rest, we obtain

$$
\alpha_{\text {crit }} \sim \frac{\lambda_{A, 1}\left(1-\left(u_{A, 1}\right)_{l}^{*}\right)}{\lambda_{A, 1}\left(1-N_{A}\left(u_{A, 1}\right)_{l}^{*}\right)+\epsilon\left(N_{A}-1\right)\left(u_{B, 1}\right)_{l}^{*}},
$$

where $\quad\left(u_{A, 1}\right)_{l}^{*}=\left(u_{A, 1}\right)_{l} /\left\|\vec{u}_{A, 1}\right\|_{1} \quad$ and $\quad\left(u_{B, 1}\right)_{l}^{*}=$ $\left(u_{B, 1}\right)_{l} /\left\|\vec{u}_{B, 1}\right\|_{1}$.

The analysis of $\frac{\partial \alpha_{\text {crit }}}{\partial\left(u_{A, 1}\right)_{l}^{*}}$ implies that $\alpha_{\text {crit }}$ grows with $\left(u_{A, 1}\right)_{l}^{*}$ for $\lambda_{A, 1}>\epsilon\left(u_{B, 1}\right)_{m}^{*}$, which is always true. Furthermore, $\alpha_{\text {crit }} \rightarrow\left[1+\epsilon\left(N_{A}-1\right)\left(u_{B, 1}\right)_{m}^{*} / \lambda_{A, 1}\right]^{-1}$ when $\left(u_{A, 1}\right)_{l}^{*} \rightarrow 0$, and grows to $\infty$ when $\left(u_{A, 1}\right)_{l}^{*} \rightarrow 1 / N_{A}+\epsilon\left(u_{B, 1}\right)_{m}^{*} / \lambda_{A, 1}$. In summary, $\alpha_{\text {crit }}$ does not depend strongly on $\left(u_{A, 1}\right)_{l}^{*}$ for small values of $\left(u_{A, 1}\right)_{l}^{*}$, grows with $\left(u_{A, 1}\right)_{l}^{*}$ and tends to infinity for a finite value of $\left(u_{A, 1}\right)_{l}^{*}$. All these results agree with the numerical calculations plotted in Fig. 6 of the Supplemental Material [83]. 


\section{c. Dependency on the topology of the networks}

The strongly unequal distribution of centrality between the different nodes of scale-free networks makes the quantity $\left(u_{A, 1}\right)_{l}^{*}=\left(u_{A, 1}\right)_{l} /\left\|\vec{u}_{A, 1}\right\|_{1}$ applied to the most peripheral node (i.e., the one that will more easily connect to network B) be close to zero in general. Random (Erdős-Rényi) networks, less unbalanced than scale-free, show intermediate values of $\left(u_{A, 1}\right)_{l}^{*}$ for their most peripheral node. On the other hand, in regular networks (i.e., networks where all nodes have the same degree) and cliques (i.e., networks where all nodes are connected to the totality of the rest of the nodes), the eigenvector associated to the largest eigenvalue is $\vec{u}_{1}=$ $\left(\sqrt{N_{A}}\right)^{-1}(1,1,1 \ldots)$ and therefore $\left(u_{A, 1}\right)_{l}^{*}$ reaches the very large value of $1 / N_{A}$ for every node of network A. In summary, regarding the dependency of $\alpha_{\text {crit }}$ on $\left(u_{A, 1}\right)_{l}^{*}$ studied in the former subsection and in Fig. 6 of the Supplemental Material [83], we conclude that scale-free networks will show low values of $\alpha_{\text {crit }}$, random (Erdős-Rényi) networks intermediate values of $\alpha_{\text {crit }}$ and cliques and regular networks large values of $\alpha_{\text {crit }}$.

\section{Summary of the dependency of $\alpha_{\text {crit }}$ on the main parameters of the system}

Networks of similar strength $\left(\lambda_{1}\right)$ will connect more rarely (i.e., will have a larger $\alpha_{\text {crit }}$ associated, or in other words will need a payoff with a larger amount of self-interest) than cases in which the strong network is large and/or clearly stronger than the weak one. As increasing the size of a network in general increases its largest eigenvalue and diminishes the centrality of its most peripheral node (because the centrality is distributed among a larger number of nodes), increasing $N_{A}$ will increase the probability of connection (i.e., decrease $\alpha_{\text {crit }}$, as shown in Fig. 3). Furthermore, the central nodes of a strong network will always be more reluctant to connect than its peripheral nodes, and networks with large minimum centrality (such as regular networks or cliques) will also resist to connect more strongly than networks with small minimum centralities, such as scale-free networks. Random (Erdős-Rényi) networks will be an intermediate case between these two extremal topologies.

\section{APPENDIX F: NETWORK OF COLLABORATIONS IN GRAPHENE RESEARCH}

We retrieved from Scopus [135] the bibliographical information of all the articles published between 2007 and 2018 that contain the word graphene in the title, abstract, or keyword list. This period covers the rapid development recently experienced by that field, which rocketed from around 600 articles published in 2007 to more than 16000 in 2018. To deal with redundant author names, we relied on Scopus's author IDs. We manually checked the author IDs of the 100 most prolific researchers in the field for consistency, and found that author IDs typically correspond to single researchers and that the vast majority of publications from the same researcher were indexed under a single author ID. In total, our dataset included 104670 articles and 193675 different authors.

To assess the overall importance of each researcher, we built a global collaboration network by connecting pairs of researchers that had coauthored at least one article between 2007 and 2018, with researchers as nodes and connections weighted by the number of coauthored articles. The network provides a representation of the strength of collaborations among different authors since the field of graphene began developing. Accordingly, we quantified the researchers' global influence by means of their eigenvector centrality in the collaboration network. In this context, the eigenvector centrality provides a measure of importance that depends not only on the number of publications but also on the importance of one's collaborators.

To facilitate further analyses, we focused on the collaborations among the top 100 researchers in the global centrality rank. Of those, two researchers from the National Institute for Materials Science, each with more than 350 coauthored articles in 11 years, were identified as outliers in a preliminary inspection of collaborations among the top 100 authors. A more careful analysis revealed that those two authors contributed to a large number of worldwide experimental studies by providing high-quality graphene samples. Due to their high connectivity, the inclusion of those two authors strongly affected the topology of the collaboration network, masking any underlying modular structure that could result from geographical, strategic and or scientific affinity association among groups of researchers. To prevent this effect, we removed those two outliers from the network.

We studied the temporal evolution of the graphene coauthorship network by considering articles published in five-year windows. Because most of the top 100 researchers (75 out of 100) were already active in 2007-2012, and that timing approximately corresponds with the beginning of the "graphene boom", we chose the 2007-2012 window as the initial state of the collaboration network. To allow for consistent comparison across time windows, we excluded the 25 authors that were not already present in that initial state. In agreement with our model, the initial state of the graphene coauthorship network consisted of several disconnected or weakly connected subnetworks, each representing a community of researchers that preferentially collaborated with each other. We used Infomap [85] to identify such communities in a nonsupervised, objective way. In many cases, it was possible to correlate network communities with pioneering graphene research groups of institutes. Moreover, we checked that the modular structure of the collaboration network remained approximately stable along successive time windows, and therefore the communities detected in the initial state constitute meaningful units for the analysis of the interconnected network.

For each community, we defined the relative centrality of its members as the quotient between the centrality of a researcher and the sum of centralities of all the researchers in the community. Relative centralities were calculated using the global collaboration network (including all authors and all articles from 2007 and 2018), so they can be compared across time windows in a consistent manner. For each time window, the relative centralities of the connector nodes were used to calculate the assortativity of connections. To evaluate the significance of the trends observed in the graphene collaboration network, we performed 100 randomizations of the connector links while fixing the internal topology of each community. 
[1] T. Piketty and E. Saez, Inequality in the long run, Science 344, 838 (2014).

[2] M. Scheffer, B. van Bavel, I. A. van de Leemput, and E. H. van Nes, Inequality in nature and society, Proc. Natl. Acad. Sci. USA 114, 13154 (2017).

[3] J. P. Mackenbach, J. R. Valverde, B. Artnik, M. Bopp, H. Brønnum-Hansen, P. Deboosere, R. Kalediene, K. Kovács, M. Leinsalu et al., Trends in health inequalities in 27 European countries, Proc. Natl. Acad. Sci. USA 115, 6440 (2018).

[4] R. L. Carneiro, A theory of the origin of the state, Science 169, 733 (1970).

[5] E. M. Brumfiel and T. K. Earle, Specialization, Exchange and Complex Societies (Cambridge University Press, Cambridge, 1987).

[6] P. J. Richerson and R. Boyd, Complex societies, Hum. Nature 10, 253 (1999).

[7] D. L. Toye, The emergence of complex societies: A comparative approach, World History Connected 11, 2 (2004).

[8] W. Kets, G. Iyengar, R. Sethi, and S. Bowles, Inequality and network structure, Game Econ. Behav. 73, 215 (2011).

[9] J. L. Moreno, Who Shall Survive? A New Approach to the Problem of Human Interrelations (Nervous and Mental Disease Publishing Co., 1934).

[10] M. S. Granovetter, The strength of weak ties, Am. J. Sociol. 78, 1360 (1973).

[11] F. Lorrain and H. C. White, Structural equivalence of individuals in social networks, J. Math. Sociol. 1, 49 (1971).

[12] H. J. Leavitt, Some effects of certain communication patterns on group performance, J. Abnorm. Soc. Psychol. 46, 38 (1951).

[13] A. R. Radcliffe-Brown, Structure and Function in Primitive Society (Cohen and West, London, 1952).

[14] J. P. Boyd, The algebra of group kinship, J. Math. Psychol. 6, 139 (1969).

[15] S. P. Borgatti, A. Mehra, D. J. Brass, and G. Labianca, Network Analysis in the Social Sciences, Science 323, 892 (2009).

[16] E. D. Beinhocker, The Origin of Wealth. Evolution, Complexity, and the Radical Remakimg of Economics (Harvard Business School Press, Boston, 2006).

[17] C. A. Hidalgo and R. Hausmann, The building blocks of economic complexity, Proc. Natl. Acad. Sci. USA 106, 10570 (2009).

[18] G. Fagiolo, J. Reyes, and S. Schiavo, The evolution of the world trade web: A weighted-network analysis, J. Evol. Econ. 20, 479 (2010).

[19] D. Acemoglu and A. Ozdaglar, Opinion dynamics and learning in social networks, Dyn. Games Appl. 1, 3 (2011).

[20] D. Colander and R. Cupers, Complexity and the Art of Public Policy. Solving Society's Problems from the Bottom Up (Princeton University Press, Princeton, 2014).

[21] E. Anduiza, C. Cristancho, and J. M. Sabucedo, Mobilization through online social networks: The political protest of the indignados in Spain, Inf. Commun. Soc. 17, 750 (2014).

[22] N. Kapucu, Q. Hu, and S. Khosa, The state of network research in public administration, Admin. Soc. 49, 1087 (2017).

[23] M. R. Groenhuijzen and P. Verhagen, Testing the robustness of local network metrics in research on archeological local transport networks, Front. Digit. Humanit. 3, 6 (2016).
[24] L. Prignano, I. Morer, F. Fulminante, and S. Lozano, Modelling terrestrial route networks to understand inter-polity interactions (southern Etruria, 950-500 BC), J. Archeol. Sci. 105, 46 (2019).

[25] G. R. Salancik, Wanted: A good network theory of organization, Admin. Sci. Quart. 40, 345 (1995).

[26] B. Harrington and G. A. Fine, Where the action is: Small groups and recent developments in sociological theory, Small Gr. Res. 37, 4 (2006).

[27] P. Cairney, Complexity theory in political science and public policy, Polit. Stud. Rev. 10, 346 (2012).

[28] S. P. Borgatti, D. J. Brass, and D. S. Halgin, Social Network Research: Confusions, Criticisms, and Controversies, in Contemporary Perspectives on Organizational Social Networks, Vol. 40 (Emerald, Bingley, 2014), pp. 1-29.

[29] M. Rabin, Incorporating fairness into game theory and economics, Am. Econ. Rev. 83, 5 (1993).

[30] E. Fehr and K. M. Schmidt, A theory of fairness, competition, and cooperation, Q. J. Econ. 114, 817 (1999).

[31] H. Gintis, The Bounds of Reason: Game Theory and the Unification of the Behavioral Sciences-Revised Edition (Princeton University Press, 2014).

[32] J. Von Neumann and O. Morgenstern, Theory of Games and Economic Behavior (Princeton University Press, Princeton, 1953).

[33] L. Samuelson, Game theory in economics and beyond, J. Econ. Perspect. 30, 107 (2016).

[34] R. Axelrod, The emergence of cooperation among egoists, Am. Polit. Sci. Rev. 75, 306 (1981).

[35] D. Challet and Y. C. Zhang, Emergence of cooperation and organization in an evolutionary game, Physica A 246, 407 (1997).

[36] J. Bednar and S. Page, Can game(s) theory explain culture? The emergence of cultural behavior within multiple games, Ration. Soc. 19, 65 (2007).

[37] J. Iranzo, J. M. Buldú, and J. Aguirre, Competition among networks highlights the power of the weak, Nat. Commun. 7, 13273 (2016).

[38] F. Michor and M. A. Nowak, The good, the bad and the lonely, Nature 419, 677 (2002).

[39] G. Szabó and G. Fáth, Evolutionary games on graphs, Phys. Rep. 446(4-6), 97 (2007).

[40] A. Dreber, D. G. Rand, D. Fudenberg, and M. A. Nowak, Winners don't punish, Nature 452, 348 (2008).

[41] M. Perc and A. Szolnoki, Coevolutionary games-A mini review, Biosystems 99, 109 (2010).

[42] C. L. Apicella, F. W. Marlowe, J. H. Fowler, and N. A. Christakis, Social networks and cooperation in huntergatherers, Nature 481, 497 (2012).

[43] J. Gómez-Gardeñes, I. Reinares, A. Arenas, and L. M. Floria, Evolution of cooperation in multiplex networks, Sci. Rep. 2, 620 (2012).

[44] Z. Wang, A. Szolnoki, and M. Perc, Self-organization towards optimally interdependent networks by means of coevolution, New J. Phys. 16, 033041 (2014).

[45] J. Aguirre, R. Sevilla-Escoboza, R. Gutiérrez, D. Papo, and J. M. Buldú, Synchronization of Interconnected Networks: The Role of Connector Nodes, Phys. Rev. Lett. 112, 248701 (2014). 
[46] Z. Wang, L. Wang, A. Szolnoki, and M. Perc, Evolutionary games on multilayer networks: A colloquium, Eur. Phys. J. B 88, 124 (2015).

[47] P. Bonacich, Factoring and weighting approaches to status scores and clique identification, J. Math. Sociol. 2, 113 (1972).

[48] M. E. J. Newman, Assortative Mixing in Networks, Phys. Rev. Lett. 89, 208701 (2002).

[49] M. O. Jackson, T. Rodriguez-Barraquer, and X. Tan, Social capital and social quilts: Network patterns of favor exchange, Am. Econ. Rev. 102, 1857 (2012).

[50] A. Banerjee, A. G. Chandrasekhar, E. Duflo, and M. O. Jackson, The diffusion of microfinance, The National Bureau of Economic Research, Working Paper No. 17743 (2012).

[51] A. Banerjee, A. G. Chandrasekhar, E. Duflo, and M. O. Jackson, The diffusion of microfinance, Science 341(6144), 1236498 (2013).

[52] M. U. Ilyas and H. Radha, Identifying influential nodes in online social networks using principal component centrality, in 2011 IEEE International Conference on Communications (ICC) (IEEE, Kyoto, 2011), pp. 1-5.

[53] M. E. J. Newman, Networks: An Introduction (Oxford University Press, Inc., New York, 2010).

[54] U. Senanayake, M. Piraveenan, and A. Zomaya, The pagerank-index: Going beyond citation counts in quantifying scientific impact of researchers, PLOS ONE 10, e0134794 (2015).

[55] A. N. Langville and C. D. Meyer, Google's PageRank and Beyond: The Science of Search Engine Rankings (Princeton University Press, Princeton, 2006).

[56] J. Aguirre, J. M. Buldú, and S. C. Manrubia, Evolutionary dynamics on networks of selectively neutral genotypes: Effects of topology and sequence stability, Phys. Rev. E 80, 066112 (2009).

[57] J. Aguirre, P. Catalán, J. A. Cuesta, and S. Manrubia, On the networked architecture of genotype spaces and its critical effects on molecular evolution, Open Biol. 8, 180069 (2018).

[58] G. Lohmann, D. S. Margulies, A. Horstmann, B. Pleger, J. Lepsien, D. Goldhahn, H. Schloegl, M. Stumvoll, A. Villringer, and R. Turner, Eigenvector centrality mapping for analyzing connectivity patterns in fMRI data of the human brain, PLOS ONE 5, e10232 (2010).

[59] S. P. Borgatti, Centrality and network flow, Soc. Networks 27, 55 (2005).

[60] M. J. Zaki and W. Meira, Data Mining and Analysis: Fundamental Concepts and Algorithms (Cambridge University Press, New York, 2014).

[61] B. R. Craig, M. Koetter, and U. Krüger, Interbank lending and distress: Observables, unobservables, and network structure, Deutsche Bundesbank Discussion Paper No. 18/2014 (2014).

[62] M. D. König and S. Battiston, From graph theory to models of economic networks. a tutorial, in Networks, Topology and Dynamics: Theory and Applications to Economics and Social Systems, edited by A. K. Naimzada, S. Stefani, and A. Torriero (Springer, Berlin, 2009), pp. 23-63.

[63] P. Glasserman and H. P. Young, Contagion in financial networks, J. Econ. Lit. 54, 779 (2016).

[64] J. M. Buldú, F. Pablo-Martí, and J. Aguirre, Taming outof-equilibrium dynamics on interconnected networks, Nat. Commun. 10, 5314 (2019).
[65] V. Moosavi and G. Isacchini, A Markovian model of evolving world input-output network, PLOS ONE 12, e0186746 (2017).

[66] S. Battiston, M. Puliga, R. Kaushikand, P. Tasca, and G. Caldarelli, DebtRank: Too central to fail? Financial networks, the FED and systemic risk, Sci. Rep. 2, 541 (2012).

[67] A. Alter, B. R. Craig, and P. Raupach, Centrality-based capital allocations, Int. J. Cent. Bank 11(3), 329 (2015).

[68] G. Covi, M. Z. Gorpe, and C. Kok, Comap: Mapping contagion in the euro area banking sector, European Central Bank Working Paper Series No. 2224 (2019).

[69] M. D. König, S. Battiston, M. Napoletano, and F. Schweitzer, On algebraic graph theory and the dynamics of innovation networks, Netw. Heterog. Media 3, 201 (2008).

[70] M. D. König and C. J. Tessone, Network evolution based on centrality, Phys. Rev. E 84, 056108 (2011).

[71] M. D. König, S. Battiston, M. Napoletano, and F. Schweitzer, Recombinant knowledge and the evolution of innovation networks, J. Econ. Behav. Organ. 79, 145 (2011).

[72] J. Q. Dong and C.-H. Yang, Being central is a double-edged sword: Knowledge network centrality and new product development in U.S. pharmaceutical industry, Technol. Forecast. Soc. 113, 379 (2016).

[73] N. Salman and A.-L. Saives, Indirect networks: An intangible resource for biotechnology innovation, R\&D Manage. 35, 203 (2005).

[74] L. Tahmooresnejad and C. Beaudry, The importance of collaborative networks in Canadian scientific research, Ind. Innov. 25, 990 (2018).

[75] M. D. König, C. J. Tessone, and Y. Zenou, Nestedness in networks: A theoretical model and some applications, Theor. Econ. 9, 695 (2014).

[76] J. Aguirre, D. Papo, and J. M. Buldú, Successful strategies for competing networks, Nat. Phys. 9, 230 (2013).

[77] S. Boccaletti, G. Bianconi, R. Criado, C.I. del Genio, J. Gómez-Gardeñes, M. Romance, I. Sendiña-Nadal, Z. Wang, and M. Zanin, The structure and dynamics of multilayer networks, Phys. Rep. 544, 1 (2014).

[78] M. J. Osborne and A. Rubinstein, A Course in Game Theory (MIT Press, Cambridge, MA, 1994).

[79] K. Anand, P. Gai, S. Kapadia, S. Brennan, and M. Willison, A network model of financial system resilience, J. Econ. Behav. Organ. 85, 219 (2013).

[80] C. Minoiu and J. A. Reyes, A network analysis of global banking: 1978-2010, J. Financ. Stabil. 9, 168 (2013).

[81] D. Acemoglu, A. Ozdaglar, and A. Tahbaz-Salehi, Systemic risk and stability in financial networks, Am. Econ. Rev. 105, 564 (2015).

[82] S. Battiston, J. D. Farmer, A. Flache, D. Garlaschelli, A. G. Haldane, H. Heesterbeek, C. Hommes, C. Jaeger, R. May, and M. Scheffer, Complexity theory and financial regulation, Science 351, 818 (2016).

[83] See Supplemental Material at http://link.aps.org/ supplemental/10.1103/PhysRevResearch.2.043352 for several numerical simulations of the emerging society game (Supplemental Figs. 1-5), for the dependency of the critical self-interest parameter $\alpha_{\text {crit }}$ on the centrality of the connector nodes of the strong network for different networks (Supplemental Fig. 6), and for a plot of the association between the h-index and the centrality of the 
top 100 researchers in the graphene collaboration network (Supplemental Fig. 7).

[84] S. Grauwin, E. Bertin, R. Lemoy, and P. Jensen, Competition between collective and individual dynamics, Proc. Natl. Acad. Sci. USA 106, 20622 (2009).

[85] M. Rosvall and C. T. Bergstrom, Maps of random walks on complex networks reveal community structure, Proc. Natl. Acad. Sci. USA 105, 1118 (2008).

[86] D. J. de S. Price, Little Science, Big Science (Columbia University Press, New York, 1963).

[87] S. Fortunato, C. T. Bergstrom, K. Börner, J. A. Evans, D. Helbing, S. Milojević, A. M. Petersen, F. Radicchi, R. Sinatra, B. Uzzi et al., Science of science, Science 359(6379), eaao0185 (2018).

[88] G. F. Chami, S. E. Ahnert, N. B. Kabatereine, and E. M. Tukahebwa, Social network fragmentation and community health, Proc. Natl. Acad. Sci. USA 114, E7425 (2017).

[89] C. Seierstad and T. Opsahl, For the few not the many? The effect of affirmative action on presence, prominence, and social capital of women directors in Norway, Scand. J. Manag. 27, 44 (2010).

[90] R. B. Cialdini and M. R. Trost, Social influence: Social norms, conformity, and compliance, in The Handbook of Social Psychology (McGraw-Hill, 1998), pp. 151-192.

[91] V. Griskevicius, N. J. Goldstein, C. R. Mortensen, R. B. Cialdini, and D. T. Kenrick, Going along versus going alone: When fundamental motives facilitate strategic (non)conformity, J. Pers. Soc. Psychol. 91, 281 (2006).

[92] J. Henrich and R. Boyd, Why people punish defectors: Weak conformist transmission can stabilize costly enforcement of norms in cooperative dilemmas, J. Theor. Biol. 208, 79 (2001).

[93] R. L. Kendal, I. Coolen, and K. N. Laland, The role of conformity in foraging when personal and social information conflict, Behav. Ecol. 15, 269 (2004).

[94] M. Kalmijn, Intermarriage and homogamy: Causes, patterns, trends, Annu. Rev. of Sociol. 24, 395 (1998).

[95] A. J. Kaba, Inter-ethnic/interracial romantic relationships in the United States: Factors responsible for the low rates of marriages between blacks and whites, Sociol. Mind 01, 121 (2011).

[96] K. K. Denton, Y. Ram, U. Liberman, and M. W. Feldman, Cultural evolution of conformity and anticonformity, Proc. Natl. Acad. Sci. USA 117, 13603 (2020).

[97] R. A. Johnson, Religious Assortative Marriage in the United States (Academic Press, New York, 1980).

[98] Z. Qian, Who intermarries? Education, nativity, region, and interracial marriage, 1980 and 1990, J. Comp. Fam. Stud. 30, 579 (1999).

[99] K. Davis, Intermarriage in caste societies, Am. Anthropol. 43, 376 (1941).

[100] J. F. Padgett, Open elite? Social mobility, marriage, and family in Florence, 1282-1494, Renaissance Quart. 63, 357 (2010).

[101] I. Briz i Godino, J. I. Santos, J. M. Galán, J. Caro, M. Álvarez, and D. Zurro, Social cooperation and resource management dynamics among late hunter-fisher-gatherer societies in Tierra del Fuego (South America), J. Archaeol. Method. Th. 21, 343 (2014).

[102] W. Wang, The rise of intermarriage, Pew Research Center, (Washington DC, 2012) [WWW document].
[103] P. D. Ellis, Social ties and international entrepreneurship: Opportunities and constraints affecting firm internationalization, J. Int. Bus. Stud. 42, 99 (2011).

[104] B. Neyt, S. Vandenbulcke, and S. Baert, Education level and mating success: Undercover on Tinder (IZA Discussion Papers, Bonn, 2018).

[105] M. R. Robinson, A. Kleinman, M. Graff, A. A. E. Vinkhuyzen, D. Couper, M. B. Miller, W. J. Peyrot, A. Abdellaoui, B. P. Zietsch, I. M. Nolte et al., Genetic evidence of assortative mating in humans, Nat. Hum. Behav. 1, 1 (2017).

[106] M. T. Rivera, S. B. Soderstrom, and B. Uzzi, Dyamics of dyads in social networks: Assortative, relational, and proximity mechanism, Annu. Rev. Sociol. 36, 91 (2010).

[107] D. T. Lichter and Z. Qian, The study of assortative mating: Theory, data, and analysis, in Analytical Family Demography (Springer, Cham., 2019), pp. 303-337.

[108] G. S. Becker, A theory of marriage: Part I, J. Polit. Econ. 81, 813 (1973).

[109] H. Liu and J. Lu, Measuring the degree of assortative mating, Econ. Lett. 92, 317 (2006).

[110] Y. Sugita, K. Teshima, and E. Seira, Assortative matching of exporters and importers (Institute of Developing Economies (IDE), Chiba, 2016).

[111] P. Antràs, L. Garicano, and E. Rossi-Hansberg, Offshoring in a knowledge economy, Q. J. Econ. 121, 31 (2006).

[112] R. A. Bentley, P. Bickle, L. Fibiger, G. M. Nowell, C. W. Dale, R. E. M. Hedges, J. Hamilton, J. Wahl, M. Francken, G. Grupe et al., Community differentiation and kinship among Europe's first farmers, Proc. Natl. Acad. Sci. USA 109, 9326 (2012), https://www.pnas.org/content/109/24/9326.full.pdf.

[113] S. Bowles, The emergence of inequality and hierarchy: A network explanation (presentation at the January 2009 meetings of the American Economic Association, tuvalu.santafe.edu/ bowles/NetworkExplanation.pdf, San Francisco, 2009).

[114] N. Creanza, O. Kolodny, and M. W. Feldman, Cultural evolutionary theory: How culture evolves and why it matters, Proc. Natl. Acad. Sci. USA 114, 7782 (2017).

[115] A. Alland, Cultural evolution: the Darwinian model. Soc. Biol. 19, 227 (1972).

[116] M. W. Feldman and L. L. Cavalli-Sforza, Cultural and biological evolutionary processes, selection for a trait under complex transmission, Theor. Popul. Biol. 9, 238 (1976).

[117] N. Creanza, L. Fogarty, and M. W. Feldman, Models of cultural niche construction with selection and assortative mating, PLOS ONE 7(8), e42744 (2012).

[118] F. J. Odling-Smee, K. N. Laland, and M. W. Feldman, Niche Construction: The Neglected Process in Evolution (Princeton University Press, Princeton, 2013).

[119] K. N. Laland, J. Odling-Smee, and M. W. Feldman, Niche construction, biological evolution, and cultural change, Behav. Brain. Sci. 23, 131 (2000).

[120] M. C. Keller, C. E. Garver-Apgar, M. J. Wright, N. G. Martin, R. P. Corley, M. C. Stallings, J. K. Hewitt, and B. P. Zietsch, The genetic correlation between height and IQ: Shared genes or assortative mating? PLOS Genet. 9(4), e1003451 (2013).

[121] B. W. Domingue, J. Fletcher, D. Conley, and J. D. Boardman, Genetic and educational assortative mating among US adults, Proc. Natl. Acad. Sci. USA 111, 7996 (2014) 
[122] I. Eshel and L. L. Cavalli-Sforza, Assortment of encounters and evolution of cooperativeness, Proc. Natl. Acad. Sci. USA 79, 1331 (1982).

[123] N. Creanza and M. W. Feldman, Complexity in models of cultural niche construction with selection and homophily, Proc. Natl. Acad. Sci. USA 111, 10830 (2014).

[124] D. S. Falconer and T. F. C. Mackay, Introduction to Quantitative Genetics (Pearson Education, Harlow, 1996).

[125] R. A. Fisher, XV.-The correlation between relatives on the supposition of Mendelian inheritance, T. Roy. Soc. Edin. 52, 399 (1919).

[126] A. C. Heath, L. J. Eaves, W. E. Nance, and L. A. Corey, Social inequality and assortative mating: Cause or consequence? Behav. Genet. 17, 9 (1987).

[127] W. E. Nance, Relevance of the marriages of twins to the causal analysis of nonrandom mating, Prog. Clin. Biol. Res., 69, 61 (1981).

[128] B. Laeng, R. Mathisen, and J. A. Johnsen, Why do blue-eyed men prefer women with the same eye color? Behav. Ecol. Sociobiol. 61, 371 (2007).

[129] C. R. Schwartz, Trends and variation in assortative mating: Causes and consequences, Annu. Rev. Sociol. 39, 451 (2013).
[130] H. Guclu, J. Read, C. J. Vukotich, Jr., D. D. Galloway, H. Gao, J. J. Rainey, A. Uzicanin, S. M. Zimmer, and D. A. T. Cummings, Social contact networks and mixing among students in K-12 schools in Pittsburgh, PA, PLOS ONE 11, e0151139 (2016).

[131] K.M. Harris, C. T. Halpern, E. Whitsel, J. Hussey, J. Tabor, P. Entzel, and J. R. Udry, The national longitudinal study of adolescent to adult health: Research design, (Carolina Population Center, University of North Carolina at Chapel Hill, Chapel Hill, 2009) [WWW document].

[132] W. W. Powell, K. W. Koput, D. R. White, and J. Owen-Smith, Network dynamics and field evolution: The growth of interorganizational collaboration in the life sciences, Am. J. Soc. 110, 1132 (2005)

[133] D. G. Rand, S. Arbesman, and N. A. Christakis, Dynamic social networks promote cooperation in experiments with humans, Proc. Natl. Acad. Sci. USA 108, 19193 (2011).

[134] H. Shirado, F. Fu, J. H. Fowler, and N. A. Christakis, Quality versus quantity of social ties in experimental cooperative networks, Nat. Commun. 4, 2814 (2013).

[135] https://www.scopus.com, accessed November 27, 2018. 